Samovar: a thermomechanical code for modeling of geodynamic processes in the lithosphere-application to basin evolution

Elesin, Y; Gerya, T; Artemieva, Irina; Thybo, Hans

Published in:

Arabian Journal of Geosciences

DOI:

10.1007/s12517-010-0215-1

Publication date:

2010

Document version

Early version, also known as pre-print

Citation for published version (APA):

Elesin, Y., Gerya, T., Artemieva, I., \& Thybo, H. (2010). Samovar: a thermomechanical code for modeling of geodynamic processes in the lithosphere-application to basin evolution. Arabian Journal of Geosciences, 3(4), 477-497. https://doi.org/10.1007/s12517-010-0215-1 


\title{
Samovar: a thermomechanical code for modeling of geodynamic processes in the lithosphere-application to basin evolution
}

\author{
Yuriy Elesin · Taras Gerya · Irina M. Artemieva • \\ Hans Thybo
}

Received: 27 February 2010 / Accepted: 4 October 2010 / Published online: 11 November 2010

(C) Saudi Society for Geosciences 2010

\begin{abstract}
We present a new 2D finite difference code, Samovar, for high-resolution numerical modeling of complex geodynamic processes. Examples are collision of lithospheric plates (including mountain building and subduction) and lithosphere extension (including formation of sedimentary basins, regions of extended crust, and rift zones). The code models deformation of the lithosphere with viscoelastoplastic rheology, including erosion/sedimentation processes and formation of shear zones in areas of high stresses. It also models steady-state and transient conductive and advective thermal processes including partial melting and magma transport in the lithosphere. The thermal and mechanical parts of the code are tested for a series of physical problems with analytical solutions. We apply the code to geodynamic modeling by examining numerically the processes of lithosphere extension and basin formation. The results are directly applicable to the Basin and Range province, western USA, and demonstrate the roles of crust-mantle coupling, preexisting weakness zones, and erosion rate on the evolutionary trends of extending continental regions. Modeling of basin evolution indicates a critical role of syn-rift sedimentation
\end{abstract}

\footnotetext{
Y. Elesin $(\varangle) \cdot$ I. M. Artemieva $\cdot$ H. Thybo

Department of Geography and Geology,

University of Copenhagen, Copenhagen 1350, Denmark

e-mail: ye@geol.ku.dk

T. Gerya

Geophysical Fluid Dynamics Group,

Institute of Geophysics, Department of Geosciences,

Swiss Federal Institute of Technology (ETH-Zurich),

8093 Zurich, Switzerland
}

on the basin depth and a governing role of Peierls deformation in cold lithospheric mantle. While the former may increase basin depth by $50 \%$, the latter limits the depth of rift basins by preventing faulting in the subcrustal lithosphere.

Keywords Geodynamic modeling • Viscoelastoplastic rheology $\cdot$ Peierls creep $\cdot$ Basin analysis

\section{Introduction}

Modeling of complex geodynamic processes in the lithosphere, such as magmatic intrusions into the crust and basin formation, requires consideration of three different types of material deformation: viscous, elastic, and plastic. Viscous behavior is suitable for modeling sublithospheric mantle and magma flow, and almost all geological materials behave viscous on a long-time scale. The elastic approximation is used for modeling flexure processes such as lithospheric bending. Plastic deformation represents brittle behavior of a material that fails when stresses exceed the maximum sustainable stress value (yield stress). We develop a new 2D code for geodynamic modeling, Samovar, which incorporates all three types of deformation. Additionally, it handles complex erosional processes, which have received substantial attention recently, as these processes can significantly modify deformation of the lithosphere (e.g., Avouac and Burov 1996; Burov and Poliakov 2001; Garcia-Castellanos et al. 2003; Fischer et al. 2004; Pysklywec 2006; Burov and Toussaint 2007; Kaus et al. 2008; Selzer et al. 2008; Bialas and Buck 2009).

The governing equations in Samovar determine material velocity, pressure, and temperature in the system 
at any given time based on a series of initial and boundary conditions:

- Stokes equation represents conservation of momentum. Although Stokes equation traditionally is used for modeling of viscous flow, it can also be adapted to complex rheology to account for elastic and plastic material behavior.

- Mass conservation equation. For incompressible material, this equation reduces to volume conservation. Stokes and mass conservation equations are used to determine the velocity and pressure distribution within the modeling area at any given time.

- Conservation of energy is used to calculate the temperature distribution within the modeling area based on the initial temperature distribution, heat sources, and boundary conditions.

Samovar uses finite difference discretization of the governing equations to calculate the velocity and temperature fields inside the modeling domain. The computational grid is regular, fixed, and cartesian, with the variables defined at different (staggered) nodes. Different materials are implemented initially as particles (markers) with different physical properties (density, viscosity, thermal conductivity, etc.), which are calculated at these markers and then interpolated onto the computational grid. Once a solution of the governing equations is found on the numerical grid, the parameters defining viscous, elastic, and plastic material behavior (stresses and strain rates) are recalculated. These parameters are interpolated onto the markers, which move according to the local velocity field and chosen time step. This cycle is repeated successively.

The "particle in cell method" (Harlow and Welch 1965) is efficient for solving problems with high strains in the system: Physical parameters and material properties follow the deforming material (particles), so that a computationally efficient, regular grid can be retained, while material deformation is tracked by markers. Solving the governing equations in the material reference frame significantly simplifies calculations. The drawbacks of using markers are the requirement of additional memory storage and increased computational time (the latter, however, is very small compare to the time spent on solving the governing equations).

Samovar uses a direct solver-Pardiso (Schenk and Gartner 2004)—for Stokes and continuity equations, allowing to use numerical grids with up to $2,000 \times 400$ grid nodes (higher spatial resolutions might be possible, but the overall computational time becomes very large, and therefore, it was never tested in Samovar). The heat conduction equation is solved explicitly for small time steps and using the direct solver whenever the time step is large. Samovar was developed as a robust high-resolution 2D tool for geodynamic modeling of lithospheric processes with the primary application to basin formation.

This paper briefly outline the features of other codes for geodynamic modeling of lithospheric processes and describes the mathematical foundation of the physical processes involved in the modeling. We also outline the main steps of the calculation scheme of Samovar. A series of benchmark tests demonstrate the ability of Samovar to handle viscous, elastic, and plastic deformation as well as energy conservation equation. Finally, we demonstrate the application of the code to studies of basin formation with emphasis on different erosional regimes and the implication of Peierls creep mechanism for deformation in the upper lithospheric mantle.

\section{Comparison with other thermomechanical codes}

Several thermomechanical codes have been developed recently to solve geodynamic problems within lithosphere and upper mantle (Table 1). They include the following:

Paravoz (Poliakov et al. 1993) This code originates from a Fast Lagrangian Analysis of Continua (FLAC) type code (Cundall 1989). It uses a mixed finite difference/finite element scheme. The Lagrangian mesh of Paravoz is composed of quadrilateral elements subdivided into two couples of triangular subelements with trilinear shape functions. It is a large-strain, fully explicit, time-marching algorithm. It locally solves the Newtonian equations of motion in a continuum

Table 1 Numerical codes for modeling geodynamic problems within lithosphere and upper mantle

\begin{tabular}{lllll}
\hline Code & Geometry & Method & Markers & Reference \\
\hline Paravoz & 2D & FEM $^{\text {a }}$ & - & {$[1]$} \\
Ellipsis & 2D & FEM & $\checkmark$ & {$[2]$} \\
Underworld & 2D/3D & FEM & $\checkmark$ & {$[3]$} \\
SloMo & 2D & FEM & $\checkmark$ & {$[4]$} \\
I2ELVIS & 2D & FDM & $\checkmark$ & {$[5]$} \\
I3ELVIS & 3D & FDM & $\checkmark$ & {$[6]$} \\
LAPEX-2D & 2D & FEM $^{\text {a }}$ & $\checkmark$ & {$[7]$} \\
SLIM3D & 2D/3D & ALE & $\checkmark$ & {$[8]$} \\
Samovar & 2D & FDM & $\checkmark$ & This paper \\
\hline
\end{tabular}

FEM finite element method, FDM finite difference method, ALE Arbitrary Lagrangian Eulerian, [1] Poliakov et al. (1993), [2] Moresi et al. (2003), [3] Moresi et al. (2007), [4] Kaus (2005), [5] Gerya and Burg (2007), [6] Zhu et al. (2009), [7] Babeyko and Sobolev (2008), [8] Popov and Sobolev (2008)

a Uses explicit time advancing scheme 
mechanics approximation together with the equations of heat transfer. To model viscoelastic behavior, it uses a Maxwell type description. Brittle behavior of the material is modeled using Mohr-Coulomb failure criterion.

Ellipsis (Moresi et al. 2003) In this code, the problem domain is represented by an Eulerian mesh with an embedded set of Lagrangian integration points (particles). Unknown variables are computed at the mesh nodes and the Lagrangian particles carry history variables during the deformation process. The code is based on a finite element formulation. Viscoelastic material behavior is described by the Maxwell relationship. Brittle behavior is parameterized using a non-linear effective viscosity which is introduced whenever the stress exceeds the yield value. The energy equation is solved explicitly in successive time steps. Free surface is implemented through a weak layer. "Underworld" is the new 3D version of the code (Moresi et al. 2007) based on the same principles.

SloMo (Kaus 2005) SloMo is a plane-strain finite element code which solves Stokes equations for incompressible materials. The rheology is temperaturedependent viscoelastoplastic with Mohr-Coulomb plasticity. A velocity-pressure formulation is applied on quadrilateral elements with quadratic shape functions for velocity and discontinuous linear shape functions for pressure, to avoid spurious pressures. Uzawa-type iterations are used to satisfy the incompressibility constraint.

I2ELVIS (Gerya and Yuen 2003; Gerya and Burg 2007) This code solves Stokes equation for creeping flow using the finite difference method on a Eulerian staggered grid combined with marker-in-cell technique. Material behavior is viscoelastoplastic. A weak layer is needed to simulate the free surface boundary condition. There is a 3D version of this code-I3ELVIS (Zhu et al. 2009) - which also uses finite difference discretization on a staggered grid. I3ELVIS employs viscoplastic rheological model and uses multigrid method to solve Stokes equation and Gauss-Seidel iterations for energy equation.

$L A P E X-2 D$ (Babeyko and Sobolev 2008) is a 2D thermomechanical code with finite element discretization (Babeyko and Sobolev 2008). This code is based on the computational strategy used in "Paravoz" (Poliakov et al. 1993) and follows the approach of FLAC (Cundall 1989). The code combines the explicit Lagrangian finite element algorithm of Paravoz with the particle-in-cell method. The particles are frozen in the moving Lagrangian grid and used solely to avoid numerical diffusion during remeshing of the Lagrangian grid. Material behavior is viscoelastoplastic.

SLIM3D (Popov and Sobolev 2008) is an implicit Arbitrary Lagrangian Eulerian particle-in-cell finite element code. The code is $3 \mathrm{D}$, and material behavior is described in terms of viscoelastoplastic rheology. Effects of thermal expansion and elastic compressibility are also included. The computational grid follows the free surface, so that the latter can be treated naturally, without the need of introducing a weak layer.

All the codes use finite element discretization, except for I2ELVIS, I3ELVIS, and Samovar, which use finite difference discretization. "Underworld", SLIM3D, and I3ELVIS can be used for 3D modeling but with limited spatial resolution (e.g., Moresi et al. 2007). All the codes support markers to track material properties and to prevent numerical diffusion ("Paravoz" works with a pure Lagrangian deformable mesh without markers). "Paravoz" and LAPEX-2D use explicit time integration schemes to solve the governing equations. Therefore, they are computationally cheap and memory efficient but restricted by a maximum time step. All the codes can be used to model viscoelastoplastic material behavior.

Samovar, similar to I2ELVIS, uses finite difference discretization on a staggered grid and particle-incell approach. It also includes the following features: marker splitting and merging mechanism (Moresi et al. 2003) implemented to avoid the formation of regions without markers around the computational nodes and excessive clustering of markers in other regions; diffusion and Peierls mechanisms of deformation, both important in cold regions of the lithosphere; erosion-sedimentation processes based on a combination of short-range weathering processes; and longrange fluvial transportation processes.

\section{Physical processes}

\section{Conservation equations}

Stokes equation for slow creeping flow is used as momentum equation. Gravity is the only body force in our system; therefore, Stokes equations in Cartesian coordinates takes the form:

$\frac{\partial \sigma_{i j}}{\partial x_{j}}-\frac{\partial P}{\partial x_{i}}+\rho g_{i}=0$,

where $\sigma_{i j}$ are the components of the deviatoric stress tensor, $P$ is the dynamic pressure, $\rho$ is density at a 
given point which depends on temperature, $T$ is the pressure and material composition, $g_{i}$ is the component of the gravitational acceleration in the direction of the Cartesian coordinate $x_{i}$, and $\rho g_{i}$ is the body force term.

When material temperature is below solidus $T_{\text {sol }}$ (no melting is taking place; see "Effects of melting" for details of density calculation in case of partial melting), the density in Eq. 1 is calculated using the following linear expression:

$\rho=\rho_{0}\left[1-\hat{\alpha}\left(T-T_{0}\right)+\beta\left(P-P_{0}\right)\right]$,

where $\hat{\alpha}$ is the coefficient of thermal expansion at $T$ below solidus (calculation of density for partially molten materials are described in "Effects of melting"), $\beta$ is the elastic compressibility, and $\rho_{0}$ is the reference density at $T_{0}=0^{\circ} \mathrm{C}$ and $P_{0}=10^{5} \mathrm{~Pa}$.

Stokes equation (Eq. 1) represents conservation of momentum. In order to fully describe fluid flow, the equation describing conservation of mass is added:

$\frac{\partial v_{i}}{\partial x_{i}}+\frac{1}{\rho} \frac{D \rho}{D t}=0$,

where $v_{i}$ is the component of the velocity vector in the direction of the Cartesian coordinate $x_{i}$ and $\frac{D \rho}{D t}$ is the advective time derivative of density (i.e., it describes density changes in each material point, but not density variations due to the movement of the material relative to fixed coordinate system). Using the definitions, $\alpha=$ $1 / V(\partial V / \partial T)_{P}$ and $\beta=-1 / V(\partial V / \partial P)_{T}$, one can write Eq. 3 as:

$\frac{D P}{D t}=\frac{1}{\beta}\left(\alpha \frac{D T}{D t}-\frac{\partial v_{i}}{\partial x_{i}}\right)$,

which explicitly relates the change in material volume to changes in pressure and temperature. The thermal expansion coefficient $\alpha$ in this expression is the same as $\hat{\alpha}$ in Eq. 2 when $T<T_{\text {sol }}$ and is calculated separately when melting takes place (see "Effects of melting").

The standard choices of boundary conditions are free-slip or no-slip conditions for the tangential component of velocity $v_{\tau}$ and a prescribed value for the normal component of velocity $v_{n}$. The latter may change along the boundary, but in case of a closed system with no incoming and outgoing material, it is zero all along the boundary:

$\left.v_{\tau}\right|_{\Gamma}=0$, for no slip

$\left.\frac{\partial v_{\tau}}{\partial n}\right|_{\Gamma}=0$, for free slip

$\left.v_{n}\right|_{\Gamma}=\left.f\right|_{\Gamma}$, where $n$ denotes the normal to the boundary, $\Gamma$ is the boundary of the modeling area, and $\left.f\right|_{\Gamma}$ is the function which describes how $v_{n}$ changes along the boundary.

To introduce velocity into Stokes equation (Eq. 1), the relationship between stresses and strain rates should be specified:

$\sigma_{i j}=F\left(\dot{\varepsilon}_{i j}, \eta, P, T, t, \ldots\right)$,

where $\eta$ is viscosity, $T$ is temperature, $t$ is time, and $\dot{\varepsilon}_{i j}$ is the component of deviatoric strain-rate tensor defined as:

$\dot{\varepsilon}_{i j}=\frac{1}{2}\left(\frac{\partial v_{i}}{\partial x_{j}}+\frac{\partial v_{j}}{\partial x_{i}}\right)-\frac{1}{3} \delta_{i j} \frac{\partial v_{k}}{\partial x_{k}}$

Equation 8 is called the rheological relationship, since it defines the rule by which material is deformed. The rule can be extremely complex for real materials inside the Earth. Generally, it is assumed that deformation in any material consists of three components: viscous, elastic, and brittle (plastic). More details on how it is implemented in Samovar are given in the sections below.

The last conservation law that is used in Samovar is the conservation of energy:

$\rho C_{p} \frac{D T}{D t}=\frac{\partial}{\partial x_{i}}\left(k \frac{\partial T}{\partial x_{i}}\right)+H_{a}+H_{s}+H_{r}$

where $C_{p}$ is the heat capacity at constant pressure, $k$ is the thermal conductivity, $\frac{D T}{D t}$ is advective time derivative of temperature, and $H_{a}, H_{s}, H_{r}$ are adiabatic, shear, and radiogenic heat productions per unit volume, respectively. Adiabatic and shear heating are given by the following relations:

$H_{a}=\alpha T \frac{\partial P}{\partial t}=\alpha T \frac{\partial P}{\partial x_{i}} \frac{\partial x_{i}}{\partial t}=\alpha T v_{i} \frac{\partial P}{\partial x_{i}}$,

$H_{s}=\sigma_{i j} \dot{\varepsilon}_{i j(\text { non-elastic })}$,

where $\alpha$ is the coefficient of thermal expansion and $\dot{\varepsilon}_{i j(\text { non-elastic) }}$ is the non-elastic part of the full strain rate (pure elastic deformation does not produce heat). Generally, radiogenic heat is most important in the upper crust, shear heat production is high in shear zones, and adiabatic heat is important for areas where significant and rapid pressure change takes place. Latent heat of melting in Eq. 10 is included implicitly into the $C_{p}$ coefficient (see "Effects of melting"). Boundary conditions for Eq. 10 include conditions for temperature and heat flux, and both of them can vary with time and along the boundary.

Solving Stokes, continuity, and conservation of energy equations (Eqs. 1, 3, and 10) simultaneously, one determines the velocity, pressure, and temperature fields inside the model at any given time. In application 
to real Earth materials, however, these equations are non-linear, as some of their coefficients depend on the solution of the system itself. We partially linearize some of these equations by assuming weak dependence of their parameters. In particular with a sufficiently small time step (so that $T$ and $P$ vary slowly with time), the parameters $\rho, k, \alpha, H_{s}$, and $H_{a}$ can be calculated using the values of $P, T$, and $v_{i}$ from the previous time step. Moreover, in the rheological relation (Eq. 8), $P$ and $T$ can be also used from the previous time step. Using this approach, we solve Stokes and continuity equations independently from the energy equation. The only nonlinearity in the rheological relation (the dependence of viscosity $\eta$ on the stress, see "Viscous rheology") is resolved by predicting stresses from the previous time step.

\section{Viscous rheology}

Samovar is designed specifically to model viscoelastoplastic material behavior, but it can also be used to model pure viscous, viscoelastic, or viscoplastic material deformations. This section describes how viscous rheology is implemented in the code; the subsequent sections describe how elastic and plastic formulations are introduced to Samovar.

In a Newtonian fluid, by definition, the rheological relationship (Eq. 8) between stress and strain rate is linear:

$\sigma_{i j}=2 \eta \dot{\varepsilon}_{i j}^{(v)}$,

where $\eta$ is the shear viscosity of the fluid and $\dot{\varepsilon}_{i j}^{(v)}$ denotes the viscous part of the total deviatoric strain rate. This relation provides an easy way to introduce velocity into Stokes equation (Eq. 1), which then becomes linear.

Most of Earth materials, however, behave non-linear at crustal and upper mantle conditions. Three main competing deformation mechanisms are used to describe material behavior at these conditions (Frost and Ashby 1982):

- Diffusion creep, which is a Newtonian creep, is dominant at low temperatures and low stresses. Its significance decreases with an increasing grain size.

- Dislocation creep or power-law creep is a type of deformation where strain rate increases nonlinearly with stress. This creep is dominant at high stresses and high temperatures, and it is insensitive to the grain size.

- Peierls creep or power-law breakdown. This type of deformation dominates at low temperatures and very high (>500 MPa) stresses. The rate of de- formation grows exponentially with stress, which limits the stress magnitude.

The first two mechanisms are well studied experimentally for the most common materials of the crust and upper mantle (Ranalli 1995; Karato and Wu 1993; Mei and Kohlstedt 2000a, b; Karato 2008). The importance of Peierls creep, which may control deformation in heavily stressed, cold lithosphere was studied by Kameyama et al. (1999) and Karato et al. (2001).

Diffusion and dislocation creep are independent mechanisms of deformation. As a result, their strain rates at first approximation are additive (Frost and Ashby 1982). Dislocation and Peierls creeps, on the other hand, are not independent: These processes involve the same dislocations but moving under different $P-T$ conditions. Following Frost and Ashby (1982), dislocation creep and Peierls creep can be treated as alternative mechanisms, so that only the one which results in the highest strain rate under given stress is active at the same time. In this case:

$\dot{\varepsilon}^{(v)}=\dot{\varepsilon}_{1}+\max \left(\dot{\varepsilon}_{\mathrm{n}}, \dot{\varepsilon}_{\mathrm{p}}\right)$,

where $\dot{\varepsilon}_{1}, \dot{\varepsilon}_{\mathrm{n}}$, and $\dot{\varepsilon}_{\mathrm{p}}$ are strain rates due to diffusion, dislocation, and Peierls mechanisms, respectively. A generic expression for strain rates of each of these mechanisms can be written (Karato et al. 2001) as:

$\dot{\varepsilon}^{(v)}=A \sigma^{n}\left(\frac{b}{d}\right)^{m} \exp \left[-\frac{E+P V}{R T}\left(1-\frac{\sigma}{\sigma_{p}}\right)^{q}\right]$

where $A$ is a material constant, $E$ activation energy, $V$ activation volume, $P$ pressure, $T$ temperature, $R$ gas constant, $b$ the length of Burgers vector (length of the lattice distortion in dislocations), $d$ the grain size, $m$ grain-size exponent, $n$ the stress exponent, $\sigma$ is differential stress applied to the material, $\sigma_{p}$ is the Peierls stress, and $q$ is a constant that depends on the mechanism of dislocation glide (Frost and Ashby 1982).

The parameters $A, E, V, m, n, \sigma_{p}$, and $q$ for Eq. 15 are obtained experimentally. Table 2 summarizes these flow law parameters for different materials as used in our numerical experiments. Deformation mechanism map of dry olivine (Table 2) is shown in Fig. 1. There are three stress-temperature regions with different dominating mechanism (defined as the mechanism which produces the highest strain rate).

The direct substitution of Eq. 15 into Stokes equation (Eq. 3) makes the latter non-linear. Effective viscosity for a non-Newtonian fluid is calculated from Eqs. 15 and 14 as follows:

$\eta=\frac{|\sigma|}{2\left|\dot{\varepsilon}^{(v)}\right|}$, 
Table 2 Parameters for diffusion, power-law, and Peierls creeps approximated with Eq. 15

\begin{tabular}{|c|c|c|c|c|c|c|c|c|}
\hline & $A\left(\mathrm{~Pa}^{-n} \mathrm{~s}^{-1}\right)$ & $n$ & $m$ & $E(\mathrm{~J} / \mathrm{mol})$ & $V\left(\mathrm{~m}^{3} / \mathrm{mol}\right)$ & $q$ & $\sigma_{p}(\mathrm{~Pa})$ & Ref. \\
\hline \multicolumn{9}{|l|}{ Diffusion creep ${ }^{a}$} \\
\hline Dry olivine $\mathrm{b}^{\mathrm{b}}$ & $1.1 \times 10^{5}$ & 1 & 2.5 & $3.0 \times 10^{5}$ & $6 \times 10^{-6}$ & 0 & - & {$[1]$} \\
\hline Wet olivine $^{\mathrm{b}}$ & $6.6 \times 10^{4}$ & 1 & 2.5 & $2.4 \times 10^{5}$ & $5 \times 10^{-6}$ & 0 & - & [1] \\
\hline \multicolumn{9}{|l|}{ Power-law creep } \\
\hline Wet quartzite ${ }^{b}$ & $4.0 \times 10^{-18}$ & 2.3 & 0 & $1.5 \times 10^{5}$ & - & 0 & - & [2] \\
\hline Anorthosite ${ }^{b}$ & $2.1 \times 10^{-23}$ & 3.2 & 0 & $2.4 \times 10^{5}$ & - & 0 & - & [2] \\
\hline Diabase $^{\mathrm{b}}$ & $8.0 \times 10^{-25}$ & 3.4 & 0 & $2.6 \times 10^{5}$ & - & 0 & - & [2] \\
\hline Dry olivine $^{\mathrm{b}}$ & $1.3 \times 10^{-12}$ & $3.0^{[5]}$ & 0 & $5.1 \times 10^{5[5]}$ & $14 \times 10^{-6}$ & 0 & - & {$[4,5]$} \\
\hline Wet olivine ${ }^{\mathrm{b}}$ & $2.0 \times 10^{-14}$ & $3.0^{[5]}$ & 0 & $4.1 \times 10^{5}$ & $11 \times 10^{-6}$ & 0 & - & {$[4,5]$} \\
\hline \multicolumn{9}{|l|}{ Peierls creep } \\
\hline Dry olivine $^{b}$ & $1.3 \times 10^{12}$ & 0 & 0 & $5.4 \times 10^{5}$ & 0 & 2 & $9.1 \times 10^{9}$ & [3] \\
\hline Wet olivine ${ }^{c}$ & $6.3 \times 10^{-5}$ & 2.0 & 0 & $4.7 \times 10^{5}$ & $24 \times 10^{-6}$ & 2 & $2.9 \times 10^{9}$ & [6] \\
\hline
\end{tabular}

[1] Karato et al. (1986), [2] Ranalli and Murphy (1987), [3] Evans and Goetze (1979), [4] Karato and Jung (2003), [5] Mei and Kohlstedt (2000b), [6] Katayama and Karato (2008)

${ }^{\text {a }}$ Length of Burgers vector $b$ is $5 \mathrm{~nm}$

${ }^{\mathrm{b}}$ Experiment setup: uniaxial compression

${ }^{\mathrm{c}}$ Experiment setup: simple shear

where $|\sigma|=\left(\frac{1}{2} \sigma_{i j} \sigma_{i j}\right)^{\frac{1}{2}}$ is the second deviatoric stress invariant and $\left|\dot{\varepsilon}^{(v)}\right|=\left(\frac{1}{2} \varepsilon_{i j} \varepsilon_{i j}\right)^{\frac{1}{2}}$ is the second deviatoric strain rate invariant of viscous part of deformation.

The meaning of stress $\sigma$ and strain rate $\dot{\varepsilon}$ in Eq. 15 depends on the experimental setup. In uni-axial compression tests, $\sigma$ is the differential stress and $\dot{\varepsilon}$ is the uni-axial strain rate, such that $|\sigma|=1 / \sqrt{3} \sigma$ and $|\dot{\varepsilon}|=$ $\sqrt{3} / 2 \dot{\varepsilon}$. In simple shear tests, $\sigma$ is the shear stress and $\dot{\varepsilon}$ is the shear strain rate, so that $|\sigma|=\sigma$ and $|\dot{\varepsilon}|=1 / 2 \dot{\varepsilon}$.

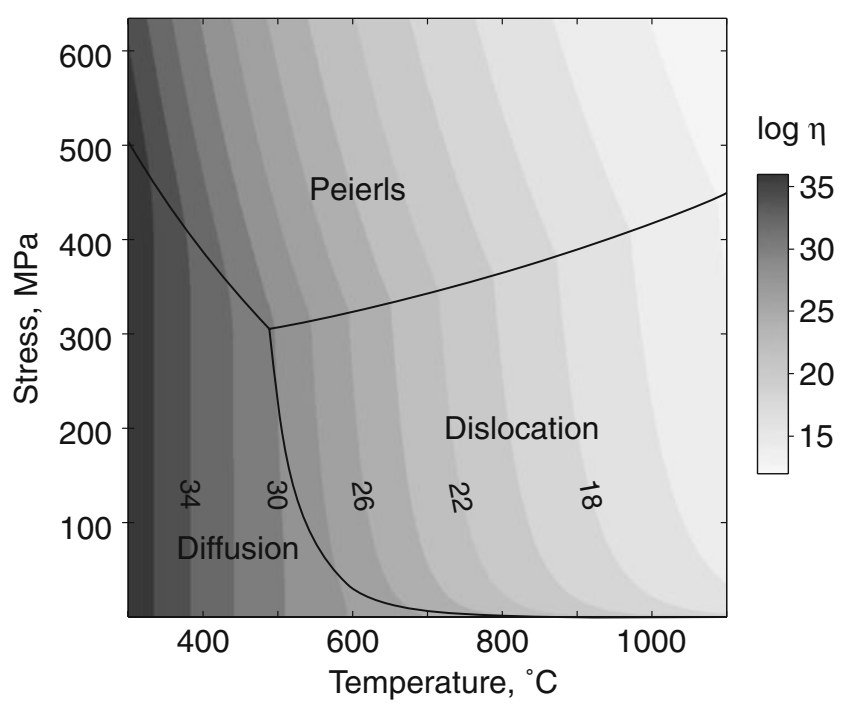

Fig. 1 Deformation mechanism map of dry olivine as functions of stress and temperature at $P=2 \mathrm{GPa}$ and grain size of $5.0 \mathrm{~mm}$
Viscoelastic formulation

Samovar uses the Maxwell formulation for viscoelastic deformation, which assumes that total strain in a viscoelastic body is the sum of the viscous and elastic strains:

$\dot{\varepsilon}_{i j}=\dot{\varepsilon}_{i j}^{(v)}+\dot{\varepsilon}_{i j}^{(e)}$,

where $\dot{\varepsilon}_{i j}^{e}$-deviatoric strain-rate tensor component due to elastic deformation and $\dot{\varepsilon}_{i j}^{v}$-viscous deviatoric strain-rate tensor component due to viscous deformation.

The viscous and elastic constitutive equations in terms of the deviatoric strain rate are:

$\sigma_{i j}=2 \eta \dot{\varepsilon}_{i j}^{(v)}$,

$\hat{\sigma}_{i j}=2 \mu \dot{\varepsilon}_{i j}^{(e)}$,

where $\mu$ is shear modulus, $\eta$ is viscosity, $\sigma$ is deviatoric stress, and $\hat{\sigma}$ is an objective material derivative of the deviatoric stress. A common choice for $\hat{\sigma}$, expressed in a difference form, is the following (Moresi et al. 2003):

$\hat{\sigma}_{i j}=\frac{\sigma_{i j}-\tilde{\sigma}_{i j}^{0}}{\Delta t}$,

where $\sigma_{i j}$ is a component of the deviatoric stress tensor at the current timestep, $\tilde{\sigma}_{i j}^{0}$ is a component of the 
deviatoric stress tensor from the previous time step corrected for advection and rotation (see numerical part for more details), and $\Delta t$ is the computational timestep. Substitution of Eqs. 18-20 into Eq. 17 gives:

$\sigma_{i j}=2 \frac{\eta \mu \Delta t}{\eta+\mu \Delta t}\left(\dot{\varepsilon}_{i j}+\frac{\tilde{\sigma}_{i j}^{0}}{2 \mu \Delta t}\right)$,

This expression is equivalent to the rheological relation for a viscous fluid (Eq. 18) if we introduce the following notations:

$\eta^{\mathrm{eff}}=\frac{\eta \mu \Delta t}{\eta+\mu \Delta t}$,

$\dot{\varepsilon}_{i j}^{\text {eff }}=\dot{\varepsilon}_{i j}+\frac{\tilde{\sigma}_{i j}^{0}}{2 \mu \Delta t}$,

with which the viscoelastic constitutive relation becomes:

$\sigma_{i j}=2 \eta^{\mathrm{eff}} \dot{\varepsilon}_{i j}^{\mathrm{eff}}$.

Using this approach, one can solve Stokes equations in the same way as for the viscous flow law. The only difference is that in this case the right-hand side of Eq. 1 will contain the term with the stresses from the previous time step according to Eq. 21.

\section{Yielding}

Rocks only show viscoelastic behavior below a critical stress value. Material fails (yields) if the stresses become higher than this critical value. Plasticity models ensure that the stress always satisfies some condition on the maximum stress which the material can support (the yield criterion). Once the yield criterion is reached, additional deformation mechanisms become active.

There are different criteria for material failure. The common choices are Tresca, Mohr-Coulomb, von Mises, and Drucker-Prager yield criteria. Samovar uses the Drucker-Prager criterion, which places an upper limit on the second invariant of the stress tensor as follows:

$|\sigma| \leq \sigma_{\text {yield }}=P \tan \phi+C$,

where $P$ is pressure and $\tan \phi$ and $C$ are the parameters to be determined experimentally. According to Byerlee's law for dry fractured rocks: $0<C<50 \mathrm{MPa}$ and $0.6<\tan \phi<0.85$ (Byerlee 1978). For wet materials, these coefficients are generally lower.

In order to satisfy condition (25), we introduce the additional plastic strain rate $\dot{\varepsilon}_{i j}^{(p)}$ in the form of the
Prandtl-Reuss flow rule (e.g., Khan and Huang 1995), which is applied when the material starts to yield $(|\sigma|=$ $\left.\sigma_{\text {yield }}\right)$ :

$\dot{\varepsilon}_{i j}=\dot{\varepsilon}_{i j}^{(v)}+\dot{\varepsilon}_{i j}^{(e)}+\dot{\varepsilon}_{i j}^{(p)}=\frac{\sigma_{i j}}{2 \eta}+\frac{\tilde{\sigma}_{i j}}{2 \mu}+\lambda \frac{\sigma_{i j}}{2|\sigma|}$,

where $\lambda$ is a parameter to be determined such that $|\sigma|=$ $\sigma_{\text {yield }}$.

Similar to the viscoelastic formulation, by expressing $\hat{\sigma}$ in a difference form (see Eq. 20) and using the following notation for effective viscosity:

$\eta_{\lambda}^{\text {eff }}=\frac{\eta_{\mathrm{vp}} \mu \Delta t}{\eta_{\mathrm{vp}}+\mu \Delta t}$,

$\eta_{\mathrm{vp}}=\frac{\eta \sigma_{\text {yield }}}{\sigma_{\text {yield }}+\lambda \eta}$

where $\eta_{\mathrm{vp}}$ is a viscosity-like parameter (Gerya and Burg 2007), one can write the viscoelastoplastic rheological relation in a form similar to the viscous flow law (see Eq. 13):

$\sigma_{i j}=2 \eta_{\lambda}^{\text {eff }} \dot{\varepsilon}_{i j}^{\text {eff }}$,

where $\dot{\varepsilon}_{i j}^{\text {eff }}$ is given by Eq. 23 .

Taking into account that $|\sigma|=\left(1 / 2 \sigma_{i j} \sigma_{i j}\right)^{1 / 2},|\dot{\varepsilon}|=$ $\left(1 / 2 \dot{\varepsilon}_{i j} \dot{\varepsilon}_{i j}\right)^{1 / 2}$ and that $|\sigma|=\sigma_{\text {yield }}$, one finds from Eq. 29:

$\eta_{\lambda}^{\text {eff }}=\frac{\sigma_{\text {yield }}}{2\left|\dot{\varepsilon}^{\text {eff }}\right|}$,

which is used to find $\eta_{\lambda}^{\text {eff }}, \eta_{\mathrm{vp}}$, and $\lambda$.

The rheological relation for viscoelastoplastic deformation in the compact form of Eq. 29 can be substituted directly into Stokes equation (1) and can be solved without additional complications in a way similar to pure viscous formulation (see "Viscous rheology"). However, just like non-linear viscosity in the viscous formulation, plasticity adds non-linearity into Stokes equation. The effective viscosity $\eta_{\lambda}^{\text {eff }}$ given by Eq. 27 depends on the parameter $\lambda$, which is equal to zero up to yielding stresses and non-zero otherwise; the latter condition can only be checked by knowing the actual stresses at the current moment of time. Therefore, the viscosity value, for which we are solving Stokes equation, depends on the solution of the equation itself and generally requires iterations (e.g., NewtonRaphson method is used in Popov and Sobolev 2008) or a good prediction of the stresses from the previous time step. Samovar uses the latter approach (see "Calculation scheme" for the details of the numerical implementation) as it significantly decreases total calculation time. The accuracy of the prediction can be controlled by adjusting the time step. Combining the prediction approach with iterations could be an 
even better approach, but it is not used in Samovar for simplicity.

\section{Plastic weakening}

The strength of the rocks can change inside the areas of plastic deformation. Samovar implements this behavior by softening or hardening of the cohesion and the friction coefficient as a function of accumulated plastic strain $\gamma_{\text {acc }}$. Following Moresi and Muhlhaus (2006), we define the rate at which plastic strain is accumulated as the difference between plastic strain rate and a healing term proportional to the viscous strain rate:

$\dot{\gamma}_{\mathrm{acc}}=\dot{\gamma}^{(p)}-\vartheta \dot{\gamma}^{(v)}$

where $\dot{\gamma}^{(p)}$ is the plastic strain rate, $\dot{\gamma}^{(v)}$ is the viscous strain rate (which is not relevant to plastic deformation and therefore assumed to heal the crack with time), and $\vartheta$ is a dimensionless healing parameter $(0<\vartheta<1)$, which characterizes how fast the material heals. The following expressions are used for plastic and viscous strain rate calculations, respectively:

$\dot{\gamma}^{(p)}=\sigma_{\text {yield }}\left(\frac{1}{\eta_{\lambda}^{\text {eff }}}-\frac{1}{\eta^{\text {eff }}}\right)$,

$\dot{\gamma}^{(v)}=\frac{|\sigma|}{\eta}$,

where $\eta_{\lambda}^{\text {eff }}$ can be found using Eq. 27 or Eq. 30 and $\eta^{\text {eff }}$ is given by Eq. 22. Combining Eqs. 31-33, we obtain the following expression for the accumulated plastic strain:

$\gamma_{\text {acc }}=\int\left(\frac{\sigma_{\text {yield }}}{\eta_{\lambda}^{\text {eff }}}-\frac{\sigma_{\text {yield }}}{\eta^{\text {eff }}}-\vartheta \frac{|\sigma|}{\eta}\right) d t$.

Equation 34 is calculated even if there is no plastic deformation. In this case, there will be only plastic healing active which decreases $\gamma_{\text {acc }}$.

Softening (hardening) of the cohesion and friction coefficient are calculated according to

$C=\alpha C_{1}+(1-\alpha) C_{0}$,

$\tan \phi=\alpha \tan \phi_{1}+(1-\alpha) \tan \phi_{0}$,

$\alpha=\min \left(1, \frac{\gamma_{\text {acc }}}{\gamma_{0}}\right)$,

where $C_{0}$ and $\tan \phi_{0}$ are cohesion and friction angle before any softening (hardening) and $C_{1}$ and $\tan \phi_{1}$ are cohesion and friction coefficients when accumulated plastic strain reaches its maximum value given by $\gamma_{0}$.
Effects of melting

Processes of melting change the physical properties of rocks in several aspects, including:

- Density, which is generally lower for molten and partially molten rock than for solid rock. The transition between solidus and liquidus cannot be described with Eq. 2 alone

- Volume normally increases while melting progresses, which is implemented through changes of the thermal expansion coefficient

- Viscosity, which decreases by several orders of magnitude when the fraction of melt in a rock is relatively high (Pinkerton and Stevenson 1992)

- Heat capacity, which may change when material transforms from solid to liquid state; additionally, melting requires latent heat, which is implemented by adjusting the heat capacity of the material

Other changes to material properties during the process of melting are of less importance for our modeling. However, if some property is expected to undergo substantial change during the melting process, it can be treated the same way as density.

The volumetric fraction of melt $M$ is calculated as (Stuwe 1995):

$M= \begin{cases}0, & \text { for } T<T_{\mathrm{s}}, \\ \frac{e^{\zeta T}-e^{\zeta T_{\mathrm{s}}}}{e^{\zeta T_{1}}-e^{\zeta T_{\mathrm{s}}}}, & \text { for } T_{\mathrm{s}} \leq T \leq T_{1}, \\ 1, & \text { for } T>T_{1} ;\end{cases}$

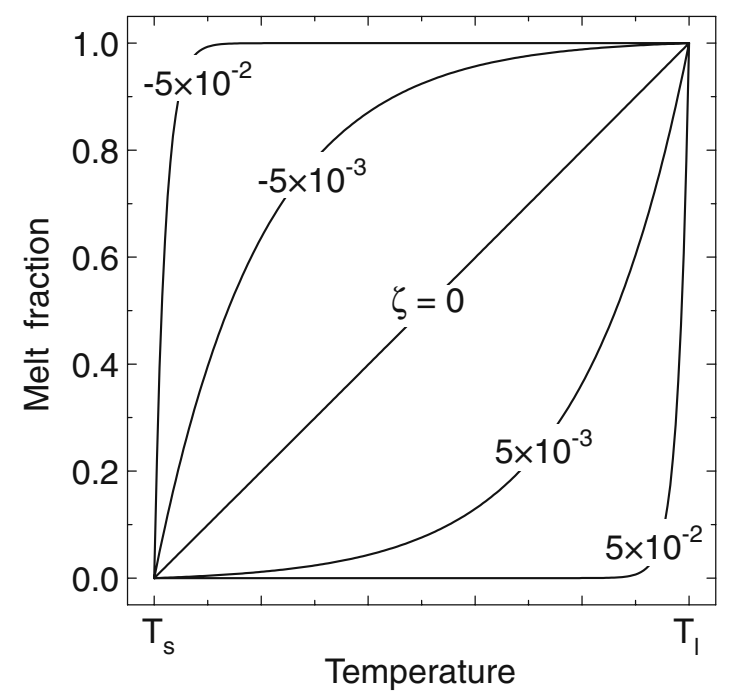

Fig. 2 Plot of the volumetric fraction of melt $M$ against temperature given by the melting model of Eq. 38. Different melting models can be controlled by varying the parameter $\zeta$, for which the diagram is contoured 
where $T_{\mathrm{s}}$ and $T_{1}$ are solidus and liquidus temperatures of the rock, respectively, and the coefficient $\zeta$, which can be chosen arbitrary, controls the rate of melt production between $T_{\mathrm{s}}$ and $T_{1}$ (Fig. 2). The melt production coefficient $\zeta$ is $\sim-0.01$ for multi-component rocks when free water is available and $\zeta>0$ for dry melting (Stuwe 1995).

Equation 2 for pressure- and temperaturedependent density calculation is only suitable for a one-phase (fully molten or solid) material. For twophase material, when temperature is between solidus $\left(T_{\mathrm{s}}\right)$ and liquidus $\left(T_{1}\right)$, density is calculated as (Bittner and Schmeling 1995):

$\rho_{\mathrm{sl}}=\rho_{\mathrm{s}}(1-M)+\rho_{\mathrm{l}} M$,

where $\rho_{\mathrm{l}}$ and $\rho_{\mathrm{s}}$ are densities of solid and molten rock, respectively, which are calculated using Eq. 2 for $T=T_{\text {sol }}$ and $T=T_{1}$, respectively. Equation 39 is used whenever $0<M<1$.

The effective viscosity $\eta$ of rocks for $0 \leq M \leq 0.1$ is calculated using Eqs. 16 and 15; when $0.1 \leq M \leq 1, \eta$ is calculated using the formula (Pinkerton and Stevenson 1992):

$\eta=\eta_{0} \exp \left[2.5+(1-M)\left(\frac{1-M}{M}\right)^{0.48}\right]$,

where parameter $\eta_{0}$ depends on rock composition, e.g., $\eta_{0}=10^{14} \mathrm{~Pa}$ s is chosen for felsic rocks and $\eta_{0}=$ $10^{13} \mathrm{Pas}$ for mafic and ultramafic rocks (Bittner and Schmeling 1995).

Latent heat of melting/crystallization is included implicitly into the effective heat capacity as (Stuwe 1995):

$C_{p}^{\mathrm{eff}}=C_{p}+Q_{L}\left(\frac{\partial M}{\partial T}\right)_{P}=C_{p}+Q_{L} \frac{\zeta e^{\zeta T}}{e^{\zeta T_{1}}-e^{\zeta T_{\mathrm{s}}}}$,

where $Q_{L}$ is latent heat of melting. This expression for $C_{p}^{\text {eff }}$ is used in energy conservation equation (Eq. 10) instead of $C_{p}$ whenever $0<M<1$.

Changes in material volume should be reflected in Eq. 4 by adjusting the coefficient of thermal expansion:

$\alpha^{\mathrm{eff}}=-\frac{1}{\rho}\left(\frac{\partial \rho}{\partial T}\right)_{P}=-\zeta \frac{\rho_{\mathrm{l}}-\rho_{\mathrm{s}}}{\rho_{\mathrm{s}}\left(e^{\zeta T_{1}}-e^{\left.\zeta T_{\mathrm{s}}\right)}\right.} e^{\zeta T}$,

This effective value of the thermal expansion coefficient $\alpha^{\text {eff }}$ is used in the continuity equation (Eq. 4) instead of standard thermal expansion coefficient $\alpha$, when $0<M<1$.
Topography

Samovar does not apply a free surface condition on the boundary between solid Earth and air. Instead, the air cover is represented by a low-density, low viscosity layer (depending on the experiment it may be desirable to include a water layer). The reason for this choice is that implementation of proper free surface boundary condition is complicated on a fixed staggered grid. This is not, however, a severe limitation: including a weak layer with density $1 \mathrm{~kg} / \mathrm{m}^{3}$ for air and $1,000 \mathrm{~kg} / \mathrm{m}^{3}$ for water, and viscosity $10^{13}-10^{14} \mathrm{~Pa}$ s appears to be a good approximation of the free surface. This approach for simulation of free surface is generally used in numerical codes with fixed calculation grid (Moresi and Muhlhaus 2006; Gerya and Burg 2007).

The movement of the free surface is based on a height function, traced according to the local velocity field using a $1 \mathrm{D}$ advection equation:

$\frac{\partial h}{\partial t}-v_{y}+v_{x}\left(\frac{\partial h}{\partial x}\right)+v^{\mathrm{es}}=0$,

where $h$ is the vertical coordinate of the surface relative to a reference level (e.g., ocean level); $v_{y}$ and $v_{x}$ are vertical and horizontal coordinates of velocity in the current point of the surface, respectively (calculated from the solution of Stokes equation); and $v^{\text {es }}$ is the erosion-sedimentation rate.

Surface processes in Phazor are simulated using three different types of erosion models:

- Short-range diffusive erosion which is the result of hillslope and stream processes

- Altitude-based flat erosion/sedimentation

- Long-range fluvial erosion represents the cumulative effect of fluvial transport

The short-range surface processes are modeled using the 1D diffusion equation (e.g., Braun and Sambridge 1997):

$v_{s}^{\mathrm{es}}=k_{s}\left(\frac{\partial^{2} h}{\partial x^{2}}\right)$,

where $v_{s}^{\text {es }}$ is the speed of short-range erosionsedimentation and $k_{s}$ is a coefficient that defines the intensity of the short-range erosional processes. In our experiments, the values of $k_{s}$ are varied from 1 to $10^{3} \mathrm{~m}^{2} /$ year, which corresponds to erosionsedimentation rates varying from 0.001 to $1 \mathrm{~mm} / \mathrm{year}$ for a relief of $1 \mathrm{~km}$ height with a wavelength of $200 \mathrm{~km}$.

The speed of the height-based flat erosion model is defined by the zero level (base level) $h_{0}$ below which 
sedimentation takes place and above which erosion is active:

$v_{t}^{\mathrm{es}}=\xi k_{l}\left(h-h_{0}\right)$,

where $v_{t}^{\text {es }}$ is the speed of long-range sedimentation; $\xi$ is dimensionless coefficient, which is calculated automatically to ensure the mass balance $(\xi=1$ in case of sedimentation, while in case of erosion it is adjusted for mass balance); and $k_{l}$ is a coefficient that defines the intensity of the flat sedimentation. A basin $1 \mathrm{~km}$ deep (relative to the $h_{0}$ level) will have sedimentation rate $1 \mathrm{~mm} /$ year for $k_{l}=10^{-6}$ year $^{-1}$. Total erosionsedimentation rate resulting from diffusive and flat erosion models is calculated as:

$v^{\mathrm{es}}=v_{t}^{\mathrm{es}}+v_{s}^{\mathrm{es}}$.

The speed of long-range fluvial erosion is given by (e.g., Kooi and Beaumont 1994; Braun and Sambridge 1997):

$\frac{\partial h}{\partial t}=\frac{Q-Q^{e}}{L_{\mathrm{e}, \mathrm{d}}}$,

where $Q$ is the sediment load, $Q^{e}$ is the local equilibrium sediment carrying capacity, and $L_{\mathrm{e}, \mathrm{d}}$ is the length scale characterizing erosion/deposition processes. The carrying capacity, $Q^{e}$, is computed as:

$Q^{e}=-K_{\mathrm{R}} q_{r} \frac{\partial h}{\partial l}$,

where $K_{\mathrm{R}}$ is a transport coefficient, $\partial h / \partial l$ is the slope in the direction of river drainage, and $q_{r}$ is the river discharge per unit width, which is related to the net precipitation rate $v_{R}$. The overall procedure for updating the topography based on the fluvial erosion model follows Braun and Sambridge (1997).

\section{Calculation scheme}

The calculation scheme (Fig. 3) consists of 12 main steps, which are shortly outlined below. The finite difference discretization of governing equations and details of interpolation of physical properties from markers to computational nodes and back are described by Gerya and Yuen (2007).

1. The value of the calculation time step $\Delta t$ is selected to ensure that the maximum displacement of the markers is smaller than the displacement step limit:

$$
\Delta t=K \min \left(\frac{\Delta x}{v_{x}^{(\max )}}, \frac{\Delta y}{v_{y}^{(\max )}}\right),
$$

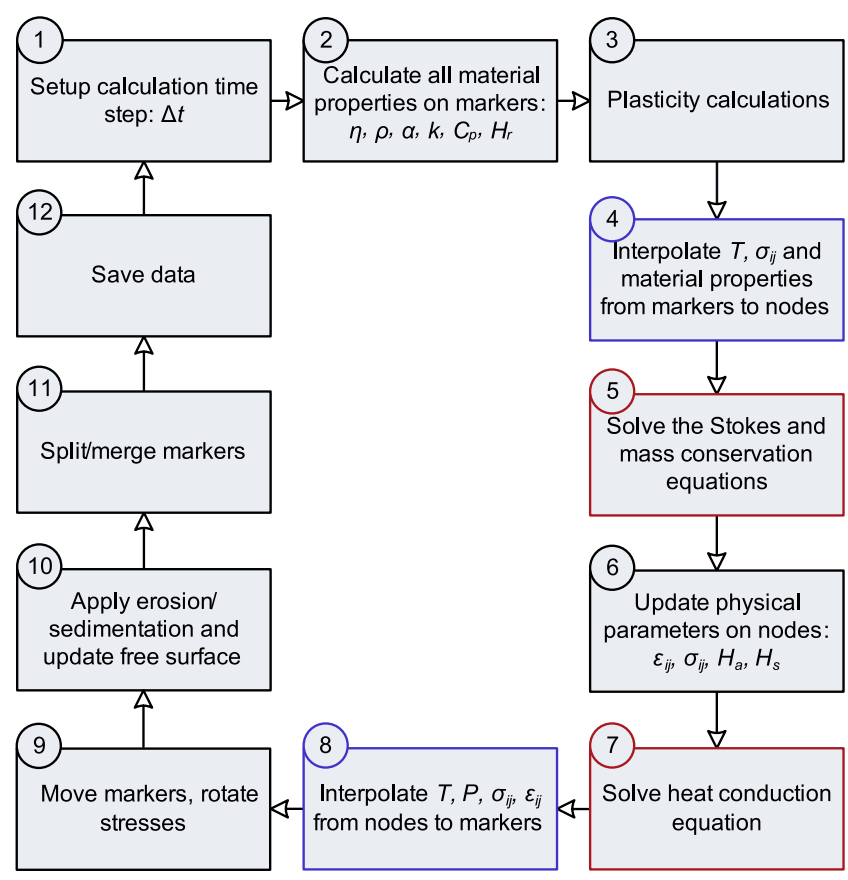

Fig. 3 Computational flowchart of Samovar

where $v_{x}^{(\max )}$ and $v_{y}^{(\max )}$ are the maximum $x$ and $y$ components of the velocity vector obtained from the previous time step, $\Delta x$ and $\Delta y$ are the grid size in $x$ - and $y$-direction, respectively, and $K$ is a dimensionless coefficient varying between 0.1 and 0.5 depending on the experiment.

2. All material properties $\left(\rho, \eta, \mu, \beta, \alpha, k, C_{p}\right.$, and $\left.H_{r}\right)$ are recalculated and assigned to markers. Some of these properties can be constant for all markers of the same material (e.g., $\mu$ and $H_{r}$ ); others depend on the physical parameters of the medium $\left(P, T, \sigma_{i j}\right)$ and are therefore recalculated using the physical parameters from the previous time step. The calculation scheme for non-linear stress-dependent viscosity $\eta$ is based on the predicted stresses from the previous time step $t^{n-1}$, which is done by solving the system of equations 16 and 21 for each marker.

3. The estimated stresses for the current time step are used in the yield criterion test (Eq. 25). If a given marker has yielded, the viscosity is recalculated using Eq. 28. The amount of yielded markers $n_{\mathrm{y}}$ is calculated and $\Delta t$ is adjusted to ensure that $n_{\mathrm{y}} \leq \alpha_{\mathrm{f}} N$, where $N$ is the total amount of markers in the model and $\alpha_{\mathrm{f}}$ is a prescribed coefficient (normally varying between $10^{-4}-10^{-2}$ ) which controls the maximum fraction of markers that can reach the yielding state in a single step, to assure that the faults will be sufficiently 
narrow (normally one to three calculation nodes across).

4. Stresses $s_{i j}$, temperature $T$ from the previous time step, and all the material properties calculated in steps 2 and 3 are interpolated from markers to the appropriate nodes of the staggered grid using a bilinear interpolation scheme.

5. Stokes and continuity equations are discretized using the finite difference method and solved using direct solver. The equations are solved in primitive variables: $P, v_{x}$, and $v_{y}$ for both compressible and incompressible formulations.

6. The following physical parameters are recalculated on the grid nodes: $\varepsilon_{i j}, \sigma_{i j}, H_{a}$, and $H_{s}$, based on the values of $v_{x}, v_{y}$, and $P$, obtained at step 5 . If the error between the calculated stresses $\sigma_{i j}$ and the stresses $\sigma_{i j}^{\mathrm{p}}$ predicted in steps 2 and 3 larger than a certain value $(1-10 \%)$, the calculation can be restarted from step 2 with decreased time step.

7. The heat conduction equation (Eq. 10) is solved explicitly for small time steps $\Delta t$ and implicitly, using direct solver, for large time steps; in both cases, finite difference discretization is used.

8. Velocity components $v_{i}$, strain rates $\varepsilon_{i j}$, pressure $P$, increments of stresses $\Delta s_{i j}$, and increment of temperature $\Delta T$ are interpolated from nodes to markers. Incremental technique (Gerya and Yuen 2003, 2007) is applied for temperature and stress interpolation to avoid numerical diffusion.

9. Stress components are rotated at each marker according to the local vorticity field. Markers are advected using an explicit Euler method for simplicity by the velocity vector interpolated at step 8 .

10. The erosion surface is moved according to the local velocity field using the $1 \mathrm{D}$ advection equation (Eq. 43) which also takes into account processes of erosion and sedimentation ("Topography"). All "weak layer" markers below the updated surface level become sediments, and all markers above the surface level become "weak layer" material.

11. By tracing the strain of each marker, we can define the moments when a given marker is too distorted (elongated), which can lead to a situation where there are very few or no markers around some calculation grid points, while there are too many markers around others. When the distortion of a given marker is too high, it is split into two markers in the direction of maximum elongation. The new markers have the same physical parameters as the original marker. If two markers of the same material are getting close to each other, they are merged into one marker and all the parameters associated with them are averaged (see Moresi et al. 2003 for more details).

12. All necessary physical parameters and material properties are saved. The computational scheme proceeds to step 1 if required.

\section{Numerical tests}

Several benchmark tests have been made to verify the applicability of Samovar to solve a range of thermomechanical problems relevant to geodynamic processes, including:

1. Instantaneous heating of a half space

2. Viscoelastic stress buildup

3. Channel flow with stress-dependent rheology

4. Couette flow with temperature-dependent viscosity

5. Couette flow with shear heating

6. Bending of an elastic plate

7. Formation of shear zones

8. Erosion model

Calculations with Samovar are compared with analytical solutions to the seven problems. The first test is designed to verify the ability of the code to solve conductive heat transfer problems. The second problem tests the buildup of the stresses in a viscoelastic Maxwell body, subjected to deformation with constant strain rate. The third problem verifies the ability of the code to deal with non-linear stress-dependent rheology. The fourth and the firth tests deal with temperature dependent rheology and shear heating. The correct solution of the sixth problem is required to ensure the ability of the code to model elastic processes of lithospheric bending. The seventh test checks the formation of shear zones in an elastoplastic layer. The angles between shear bands and the direction of the principal stress are compared to results of theoretical models of brittle deformation. The eighth example demonstrates different erosional models implemented in the code.

Instantaneous heating of a half space

A 1D half space $x \geq 0$ is subjected to an instantaneous temperature change at the initial time $t_{0}=0$ at the boundary $x=0$. The initial temperature of the medium $T_{0}=T(x, t=0)=$ const and the boundary temperature at $x=0$ is $T_{b}$. In this case, the temperature at any point of the half-space at a time $t>0$ is given by (e.g., Turcotte and Schubert 2002, page 155):

$T=T_{0}+\left(T_{b}-T_{0}\right) \operatorname{erfc}\left(\frac{x}{2 \sqrt{\kappa t}}\right)$,

where $\kappa$ is thermal diffusivity. 
In the numerical tests, we modeled a $200-\mathrm{km}$-wide ( $x$-direction) and 10-km-high (y-direction) area. The number of grid nodes in the $x$ direction was varied from 100 to 1,600 and was kept constant in the $y$ direction (ten grid nodes). The use of markers in the experiments ensures that the interpolation of temperature from nodes to markers and back does not decrease the accuracy. The total amount of markers was 100,000 and was kept constant in all experiments. Initial temperature of the medium was $0^{\circ} \mathrm{C}$, the left boundary was kept at constant temperature $1,000^{\circ} \mathrm{C}$, and the temperature at the right boundary was kept at $0^{\circ} \mathrm{C}$. Zero heat flux was prescribed at the bottom and the top boundaries. The thermal diffusivity of the material was $10^{-5} \mathrm{~m}^{2} / \mathrm{s}$. The control of the temperature variations was performed at a point $x=10 \mathrm{~km}$ from the left boundary.

The effect of grid resolution on the maximum error in the temperature calculations was examined by experiments with spatial resolution varying from five to 80 grid nodes between the heating surface and the point of measurements. The total number of grid nodes in the modeling box varied from 100 to 1,600, respectively. The initial time step was $0.1 \mathrm{Kyr}$, and it was increased at each time step by $3 \%$ until reaching a maximum time step of $100 \mathrm{Kyr}$. The total modeling time is $4 \mathrm{Myr}$. The results of the tests (Fig. 4) show that the error in the numerical solution rapidly decreases with increasing grid resolution. The minimum error with the given time step scheme is $0.3 \%$, which is reached with 20 nodes between the heating surface and the point of measurements. At higher spatial resolution, most of the error originates from the relatively long time steps and not from the spatial discretization.
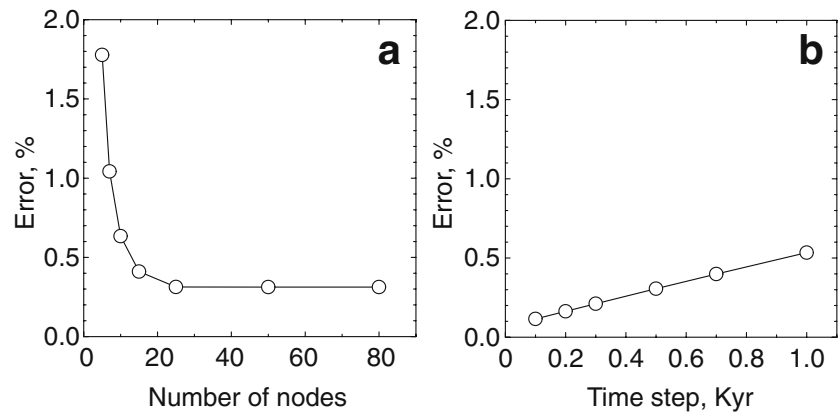

Fig. 4 Results of the numerical tests for instantaneous heating of a half-space; temperature measurements are taken $10 \mathrm{~km}$ away from the heating boundary. a Maximum relative error between the numerical and analytical solutions vs. the number of grid nodes between the heating boundary and the point of measurements. b Maximum relative error of the numerical solution vs. calculation time step. The number of grid nodes from the heating boundary to the point of "measurements" is 25
The second series of experiments was designed to analyze the effect of the time step on the accuracy of the temperature "measurements". The grid includes 25 nodes between the heating surface and the point of measurements. The time step, being constant in each experiment, varies between 0.1 and $1 \mathrm{Kyr}$ (Fig. 4). The relative error in the temperature variations decreases linearly with the length of the time step. For the given grid resolution and with a time step of less than $0.1 \mathrm{Kyr}$, the error can be lower that $0.1 \%$.

\section{Viscoelastic stress buildup}

Stress in an initially unstressed viscoelastic Maxwell body, which is subjected to deformation with constant strain rate $\dot{\varepsilon}$, grows as:

$\sigma=2 \eta \dot{\varepsilon}\left(1-e^{-\frac{\mu t}{\eta}}\right)$

where $\mu$ is shear modulus, $\eta$ is viscosity, and $t$ is time from the beginning of deformation.

To check the validity of the stress calculation in Samovar, the following numerical experiment was run. A square box with $10 \times 10 \mathrm{~km}$ of incompressible viscoelastic material with $\eta=10^{21} \mathrm{~Pa} \mathrm{~s}$ and $\mu=10^{10} \mathrm{~Pa}$ is subject to deformation with constant strain rate $\dot{\varepsilon}=$ $\dot{\varepsilon}_{x x}=10^{-13} \mathrm{~s}^{-1}$, so that normal velocity at the top and left boundaries is zero and $v=10^{-9} \mathrm{~m} / \mathrm{s}$ at the right and bottom boundaries (Fig. 5). Grid resolution is $20 \times 20$ nodes and a total of 20,000 markers is used in all numerical tests.

The numerical experiments show that the accuracy of the stress calculations does not depend on grid resolution but strongly depends on the time step (Fig. 6). The results of the numerical tests closely overlap with the analytical solution even for relatively large time steps. The error in the numerical solution linearly

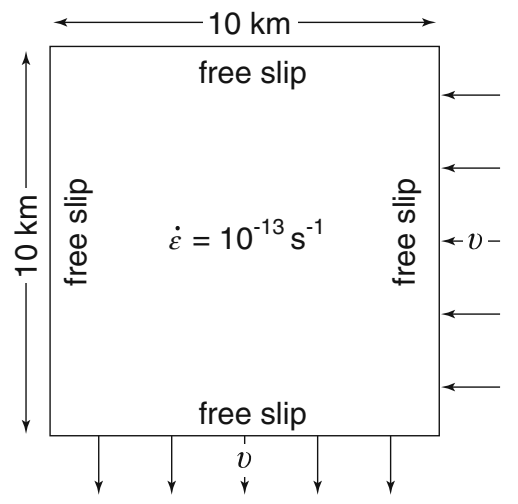

Fig. 5 Model setup for the viscoelastic stress buildup experiment. Velocity at the bottom and right boundaries is $v=10^{-9} \mathrm{~m} / \mathrm{s}$ 

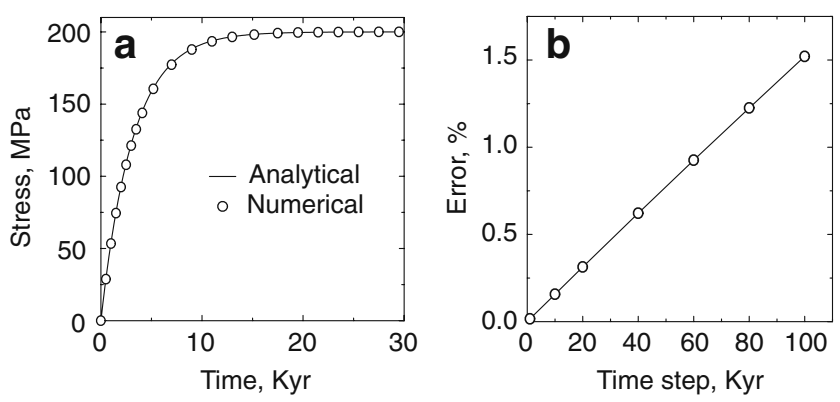

Fig. 6 The results of numerical tests of viscoelastic stress buildup. a Analytical solution of the problem compared to numerical solution with time step of 100 years. b The maximum error of the numerical solution vs. calculation time step

decreases with the time step and can be as low as $0.01 \%$ for a time step less that 1 year (Fig. 6).

\section{Channel flow with stress-dependent rheology}

We consider flow through a channel with thickness $h$ and stationary walls (no slip boundary condition), driven by a pressure gradient $\Delta p / \Delta x$. The non-linear rheology is given by the following relation:

$\tau=C\left(\frac{\partial u}{\partial y}\right)^{\frac{1}{n}}$

where $C$ is a positive constant, $\tau$ is the shear stress, $u$ is the velocity of the flow across the channel, and $n=$ $1,3,5, \ldots$ is the stress exponent.

If bottom and top boundaries of the channel are given by $y=0$ and $y=h$, the flow velocity along the channel is given by (the solution can be found by substituting Eq. 52 into Eq. 1 and integrating the latter):

$u=u_{0}\left[\left(\frac{2 y}{h}-1\right)^{n+1}-1\right]$

$u_{0}=\left(\frac{\Delta p}{\Delta x}\right)^{n} \frac{h^{n+1}}{2^{n+1}(n+1) C^{n}}$,

where $u_{0}$ is the maximum velocity along the channel (in the center).

Figure 7 compares analytical and numerical solutions for the velocity profile along the channel for $n=3$. The accuracy of the numerical models increases with increasing resolution: The maximum relative error of the numerical experiment with a grid resolution of 11 nodes along the channel is $7 \%$, while for 51 nodes it is $1.7 \%$. The maximum relative errors were obtained close to the model boundaries, where the velocity gradient is high. In the center of the channel, the error
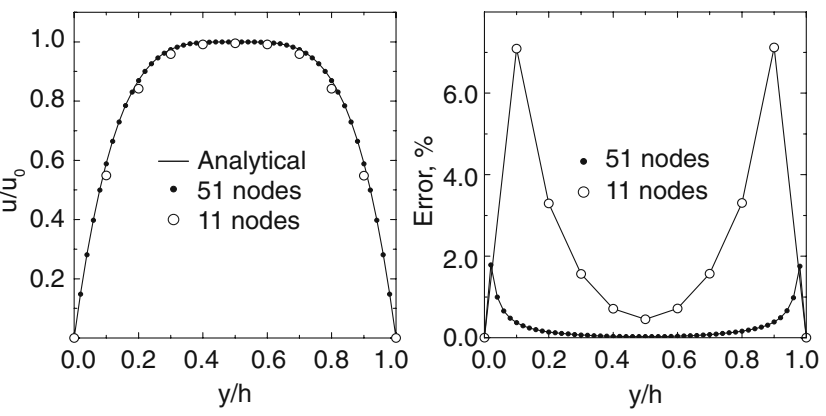

Fig. 7 The results of the numerical tests of a channel flow with stress-dependent rheology. a Comparison between analytical and numerical solutions for the velocity profile along the channel. b Numerical error in the calculated velocity along the channel. $u_{0}$ is the velocity in the center of the channel; $h$ is the thickness of the channel

in numerically calculated velocity is lower: $0.5 \%$ for 11 nodes and $0.03 \%$ when there are 51 nodes along the channel.

Couette flow with temperature-dependent viscosity

We consider Couette flow in a channel where the upper boundary $(y=h)$ moves with velocity $u_{1}$ relative to its stationary lower boundary $(y=0)$. The upper wall is maintained at temperature $T_{0}$ while the lower wall is kept at temperature $T_{1}\left(T_{1}>T_{0}\right)$. No-slip boundary condition is maintained at both walls. The viscosity of the medium in the channel is temperature dependent and given by:

$\eta=C e^{E a / R T}$,

where $C$ is a rheological constant, $E a$ is an activation energy, and $R$ is the gas constant.

Analytical solution for the velocity profile across the channel can be found in the approximation of small temperature difference between $T_{1}-T_{0}$ and $T_{0}$ (i.e., $T_{1}-T_{0} \ll T_{0}$ ) and is given by (e.g., Turcotte and Schubert 2002, page 313):

$u=u_{1} \frac{\exp \left(-\frac{E_{a}\left(T_{1}-T_{0}\right)}{R T^{2}}\left(1-\frac{y}{h}\right)\right)-1}{\exp \left(-\frac{E_{a}\left(T_{1}-T_{0}\right)}{R T^{2}}\right)-1}$.

Results of the numerical experiments with three different values of dimensionless activation energy $\left(E_{a} / R T=0,240,600\right)$ are shown in Fig. 8. The dimensionless temperature difference $\left(T_{1}-T_{0}\right) / T_{0}$ is 0.01 . Twenty-six computational nodes are used across the channel for these experiments. Good agreement 


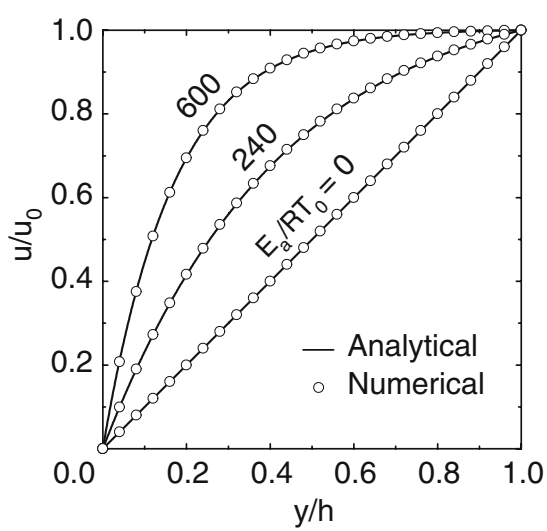

Fig. 8 Analytical and numerical solutions of velocity profile for Couette flow in a channel with linear temperature profile. The fluid's viscosity is temperature dependent and given by Eq. 55 . The dimensionless temperature difference across the channel $\left(T_{1}-T_{0}\right) / T_{0}$ is 0.01. $E_{a} / R T$ is the dimensionless activation energy parameter. The top wall $(y=h)$ is cold $\left(T=T_{0}\right)$, and the bottom wall $(y=0)$ is hot $\left(T=T_{1}\right)$. Top upper boundary moves with velocity $u_{1}$ relative to its stationary lower boundary

between analytical and numerical solutions is obtained: The maximum relative error in the velocity profile is less than $1.4 \%$.

\section{Couette flow with shear heating}

We consider a flow driven by a shear stress $\tau$ applied at the upper moving boundary $(y=h)$ of a channel with fixed lower boundary $(y=0)$. Temperature boundary conditions are:

$$
\begin{array}{ll}
T=T_{0} & \text { for } y=h, \\
\frac{d T}{d y}=0 & \text { for } y=0 .
\end{array}
$$

The viscosity of the flow is given by Eq. 55 . The analytical solution for the maximum temperature inside the channel can be found under the assumption of small temperature increase $T^{\prime}$ caused by shear heating: $T^{\prime} \ll$ $T_{0}$. If one introduces the following variables:

$\Theta=\frac{E_{a} T^{\prime}}{R T_{0}^{2}}$,

$\mathrm{Br}=\frac{\tau^{2} h^{2} E_{a} e^{-\left(E_{a} / R T_{0}\right)}}{k C R T_{0}^{2}}$,

$\phi=e^{\Theta}$,

where $\Theta$ is the dimensionless temperature and $\mathrm{Br}$ is the Brinkman number, the solution for maximum temperature inside the channel can be found solving the following transcendental equation (e.g., Turcotte and Schubert 2002, page 317):

$[\phi(1)]^{\frac{1}{2}}=\cosh \left(\frac{\operatorname{Br} \phi(1)}{2}\right)^{\frac{1}{2}}$,

where $\phi(1)$ is the exponential of the maximum temperature rise in the channel.

The following parameters were used for the numerical tests: $h=20 \mathrm{~km}, T_{0}=1,000 \mathrm{~K}, k=10 \mathrm{~W} /(\mathrm{m} \mathrm{K})$, $E_{a}=10^{6} \mathrm{~J} / \mathrm{mol}$; the speed of the upper boundary is $0.1 \mathrm{~m} /$ year; lateral resolution of the model is 11 nodes. The rheological constant $C$ was varied from $10^{-37}$ to $10^{-32} \mathrm{~Pa}$ to satisfy the condition $T^{\prime} \ll T_{0}$, for which the analytical solution was obtained.

Results of the numerical modeling are shown in Fig. 9. There is agreement between the numerical results and the analytical solution for maximum temperature change in the channel with a maximum relative error for $\Theta_{1}$ which is less then $5 \%$.

Bending of an elastic plate

The maximum deflection of an elastic rectangular beam, which bends under its own weight (uniform load), is calculated numerically and compared with the analytical solution of the Euler-Bernoulli beam equation.

"Samovar" does not include a purely elastic formulation, so the test was performed for a viscoelastic formulation with very high beam viscosity $10^{30} \mathrm{~Pa} \mathrm{~s}$ and low medium viscosity $\eta=10^{10} \mathrm{~Pa} \mathrm{~s}$ in which the beam is bending. The shear modulus of the beam is $\mu=$ $6.5 \times 10^{10} \mathrm{~Pa}$ and Poisson's ratio is 0.5 (incompressible material). Deformation of the plate can be treated as purely elastic due to its large Maxwell relaxation time

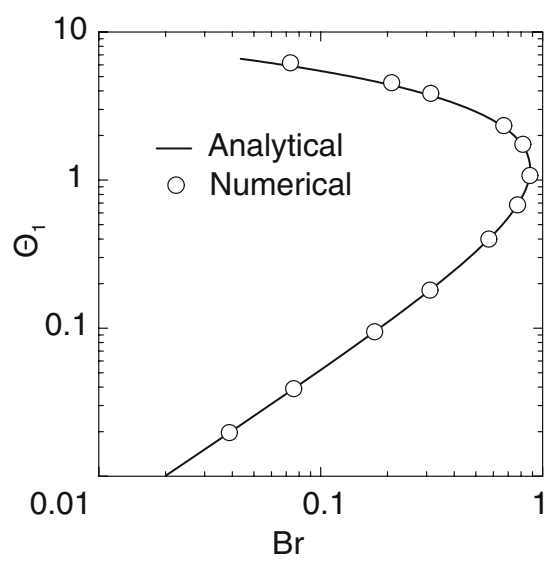

Fig. 9 Dimensionless maximum temperature change $\Theta_{1}$ (Eq. 60) vs. Brinkman number Br (Eq. 61) for the Couette flow with a temperature dependent viscosity (Eq. 55) and shear heating 


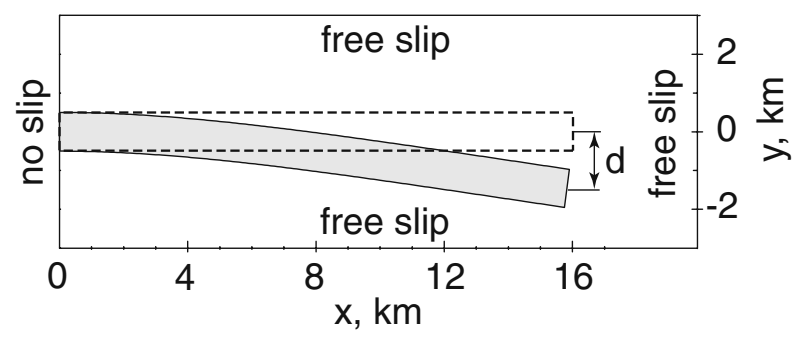

Fig. 10 The model setup for bending of an elastic plate

( $>1,000 \mathrm{Myr}$ ), which is much longer than the duration of the experiment (5 Myr). The density contrast between the medium and the beam is $10 \mathrm{~kg} / \mathrm{m}^{3}$. The number of grid nodes used in the numerical experiments varies from $18 \times 120$ to $75 \times 500$. The model setup is shown on Fig. 10.

The maximum deflection of a rectangular beam, according to Euler-Bernoulli's theory, is (e.g., Blake 1985):

$d=\frac{3 \Delta \rho g l^{4}}{2 E h^{2}}$,

where $E$ is Young's modulus, which in the case of an incompressible material is $E=3 \mu=19.5 \times 10^{10} \mathrm{~Pa}$, $h=1 \mathrm{~km}$ is the thickness of the beam, $l=16 \mathrm{~km}$ is the length of the beam, $\Delta \rho=10 \mathrm{~kg} / \mathrm{m}^{3}$ is the density contrast between the medium and the beam, and $g=$ $9.8 \mathrm{~m} / \mathrm{s}^{2}$ is the gravity acceleration.

Figure 11 illustrates the relative error in numerical experiments versus grid resolution (Eq. 63). The error rapidly decreases with increasing resolution across the beam and can be less than $5 \%$, when as little as 15 grid nodes are used across the beam. No significant increase in accuracy is obtained with a further increase of grid resolution. We attribute this fact to the presence of shear locking effect of the staggered grid (this effect

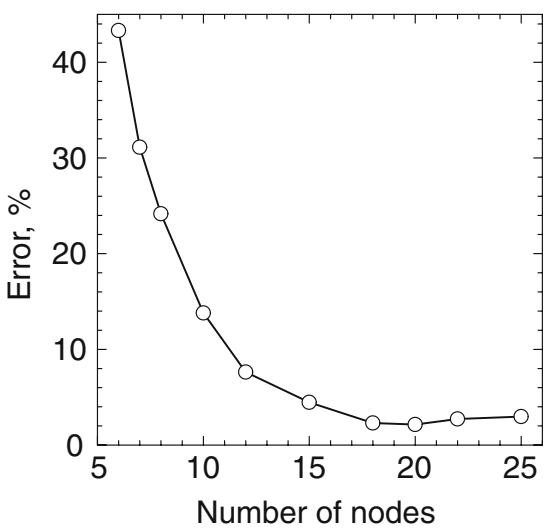

Fig. 11 Relative error in the beam deflection experiment vs. the number of grid nodes used across the beam is normally discussed in the context of finite element formulation, see, e.g., Belytschko et al. 2000).

Formation of shear zones in brittle material

We test the orientation of shear bands, which form in a brittle material, subjected to compression and extension in a box of $100 \times 12 \mathrm{~km}$. The bottom of the box $(h=10 \mathrm{~km})$ is filled with brittle material: $\rho=3,000 \mathrm{~kg} / \mathrm{m}^{3}, \mu=5 \times 10^{10} \mathrm{~Pa}, \eta=10^{25} \mathrm{~Pa} \mathrm{~s}$, cohesion $C=5 \times 10^{7} \mathrm{~Pa}$, friction coefficient $\tan \phi=$ $0.6\left(\phi=31^{\circ}\right)$, weakening coefficient $\alpha=0.6$ (Eq. 35), and maximum plastic strain $\gamma_{0}=0.2$.

A low density $\left(1 \mathrm{~kg} / \mathrm{m}^{3}\right)$, low viscosity $\left(10^{16} \mathrm{Pas}\right)$ weak layer is prescribed on top of the brittle layer to simulate a free surface. Both extension and compression experiments are performed with both side walls moving at constant speed $0.5 \mathrm{~cm} /$ year, either into the initial box for compression or outwards for extension. The resolution of the modeling area is $800 \times 96$ nodes. Material behavior is incompressible and dilatation angle $\psi=0$. Free slip boundary condition is prescribed at all the boundaries.

Results of the numerical experiments are shown in Fig. 12. The accumulated plastic strain (Eq. 34) is plotted in black so that dark zones indicate areas where failure has occurred. The dip angle of the shear bands is measured relative to the horizontal plane. For extension, the dip angle is $\sim 54^{\circ}$ and for compression it is $\sim 36^{\circ}$. Theoretical and experimental studies (Coulomb 1773; Roscoe 1970; Vermeer 1990) indicate that the dip angle typically varies between $45^{\circ}$ (Roscoe angle) and $45^{\circ}+\phi / 2$ (Coulomb angle) for extension and between $45^{\circ}$ and $45^{\circ}-\phi / 2$ for compression, so that the results of the numerical tests are in agreement with theory.
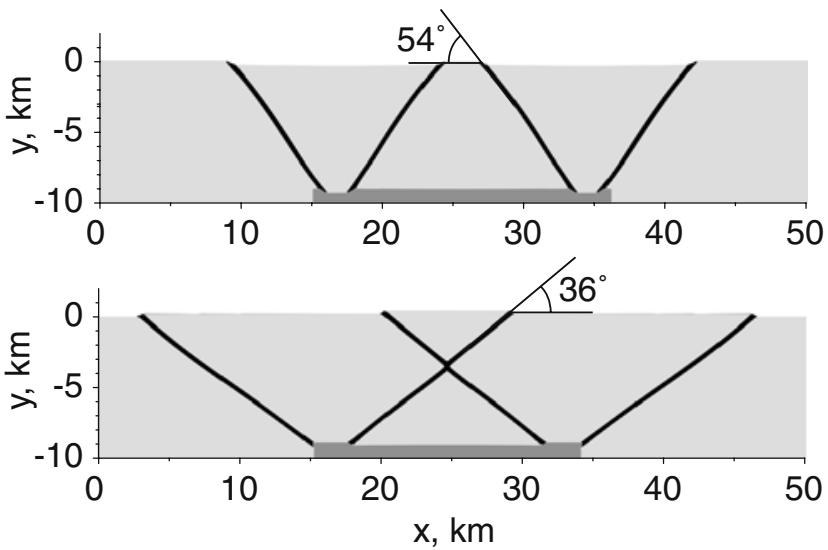

Fig. 12 Shear zone formation experiments for a extensional and b compressional settings 


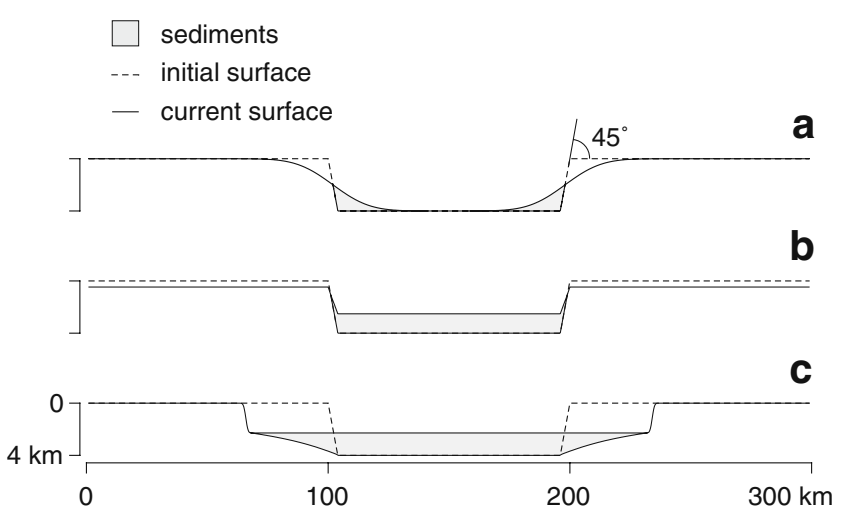

Fig. 13 Surface evolution for different erosion models: a short-range diffusive erosion, b height-based flat erosion/ sedimentation, $\mathbf{c}$ long-range fluvial erosion

\section{Erosion model}

Figure 13 illustrates the behavior of short-range and long-range erosional models ("Topography") when considered independently. The initial topography consists of a plane surface with a basin $4 \mathrm{~km}$ deep surrounded by two $45^{\circ}$ faults. The length of the modeling domain is $400 \mathrm{~km}$. No mass/energy conservation is considered in this example. The following parameters were taken for each of the three different erosion models: (a) $k_{s}=10 \mathrm{~m}^{2} /$ year for short-range diffusive erosion, (b) $k_{l}=10^{-7}$ year $^{-1}$ and $h_{0}=0 \mathrm{~m}$ for flat erosion and (c) $k_{R} v_{R}=0.1 \mathrm{~m} /$ year, $L_{e}=100 \mathrm{~km}$ for the long-range fluvial erosion model. The final surface level in Fig. 13 is shown after $7 \mathrm{Myr}$ from the beginning of each of the experiments.

Short-range diffusive erosion model (Fig. 13a) results in smoothing of topography and slope decline which is in accordance with linear diffusion model (Eq. 44) where denudation rate is proportional to the curvature of the topography. Height-based flat erosion model (Fig. 13b) results in removal of the surface from the elevated areas of the surface with a speed proportional to the surface height and sediment deposition to the lowered areas of the model with speed proportional to the depth of the surface relative to some reference level (Eq. 45). Long-range fluvial erosion (Fig. 13c) transports sediments from the slopes of the escarpments to the bottom of the basin with speed proportional to the steepness of the slopes (Eqs. 47 and 48). This results in overall broadening of the basin.

\section{Application examples}

In this section, we illustrate the applicability of Samovar to geodynamic modeling of lithospheric extension and basin formation. Both examples are based on viscoelastoplastic, stress- and temperature- dependent rheology and include processes of erosionsedimentation. The maximum resolution for the experiments is $0.5 \times 0.5 \mathrm{~km}$ on a $1,000 \times 200-\mathrm{km}$ profile. On an ordinary PC (Core 2 Duo $2.0 \mathrm{GHz}$ CPU with 4 GB of RAM), one experiments takes $\sim 20-70 \mathrm{~h}$ of CPU time.

\section{Lithospheric extension}

A uniform lithosphere is subject to extension with constant speed $1 \mathrm{~cm} /$ year. The modeling area is $1,000 \mathrm{~km}$ wide and 200 high. The model consists of several layers: a 15-km-thick weak layer, which represents air $(\rho=$ $\left.1 \mathrm{~kg} / \mathrm{m}^{3}, \eta=10^{13} \mathrm{Pas}\right)$, a $10-\mathrm{km}$-thick layer of felsic upper crust, a 30-km-thick mafic lower crust, and a mantle layer (Table 3).

Table 3 Material parameters used for modeling lithospheric extension problem

\begin{tabular}{llllll}
\hline Parameter & Sediments & Upper crust & Lower crust & Mantle \\
\hline$\rho_{0}^{[1,2,3]}$ & 2,500 & 2,700 & 2,950 & 3,350 & Units \\
$H_{r}^{[1]}$ & $1.6 \times 10^{-6}$ & $1.0 \times 10^{-6}$ & $2.5 \times 10^{-7}$ & $2.2 \times 10^{-8}$ \\
Flow law ${ }^{[6]}$ & Wet quartzite & Wet quartzite & Anorthosite & Dry olivine \\
$\mu^{[1]}$ & $1.0 \times 10^{10}$ & $2.5 \times 10^{10}$ & $3.0 \times 10^{10}$ & $6.5 \times 10^{10}$ \\
$C^{[5]}$ & 20 & 50 & 50 & 50 & $\mathrm{Wa}$ \\
$\tan \phi^{[5]}$ & 0.3 & 0.6 & 0.6 & 0.6 & 90 \\
Plastic weakening & 60 & 60 & 60 & $1.18 T+530$ & $0.73 T+1,349$ \\
$k^{[4]}$ & $0.64 T+856$ & $\frac{0.64 T+856}{T+77}$ & $\frac{T+77}{T+77}$ & $\mathrm{~W} /(\mathrm{m} \mathrm{K})$ \\
\hline
\end{tabular}

Parameters with the same value for all rocks types: $C_{p}=1,000 \mathrm{~J} / \mathrm{kg}, \alpha=3 \times 10^{-5} \mathrm{~K}^{-1}, \beta=1 \times 10^{-11} \mathrm{~Pa}^{-1}$

$\rho_{0}$ density at $T_{0}=0^{\circ} \mathrm{C}$ and $P_{0}=10^{5} \mathrm{~Pa}, H_{r}$ radioactive heat production, $\mu$ shear modulus, $C$ cohesion, $\tan \phi$ friction angle, $C_{p}$ isobaric heat capacity, $\alpha$ thermal expansion coefficient, $\beta$ compressibility, [1] Turcotte and Schubert (2002), [2] Ohtani et al. (1995), [3] Bittner and Schmeling (1995), [4] Clauser and Huenges (1995), [5] Byerlee (1978), [6] Table 2 
Fig. 14 Lithospheric deformation pattern at 12 and $32 \mathrm{Myr}$ of a 1,000-km-wide area extended with a constant speed of $1 \mathrm{~cm} /$ year. a The fault structure in the lithosphere (areas with high value of accumulated plastic strain). b Second invariant of deviatoric strain rate

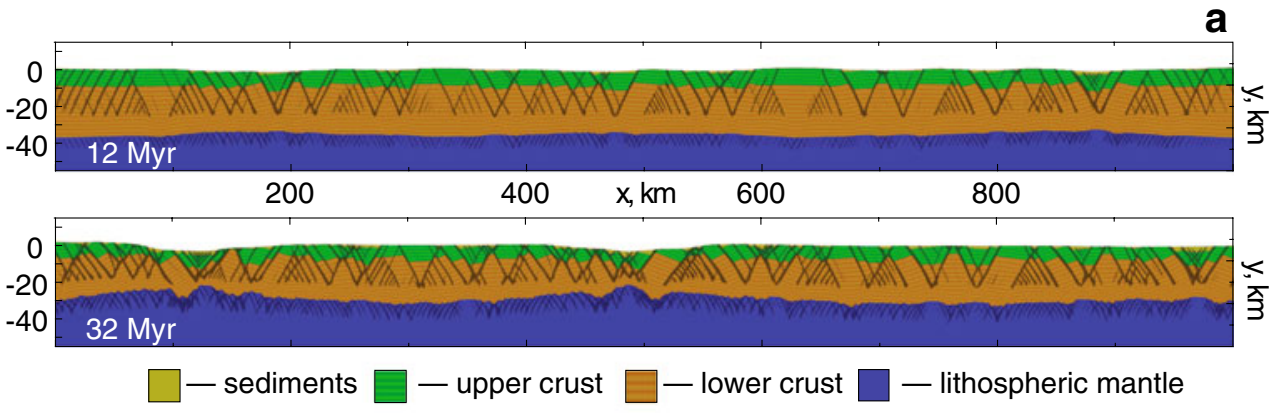

b
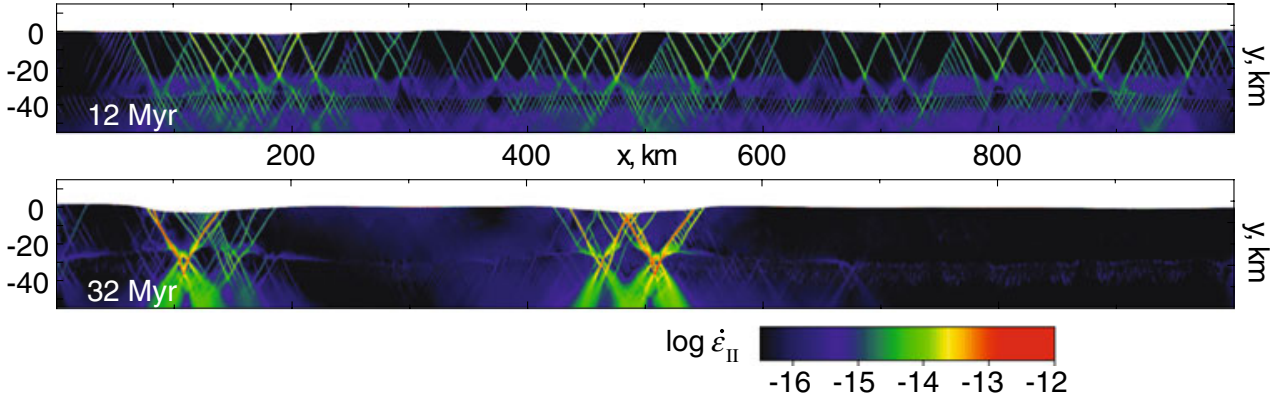

The boundary between lithospheric and asthenospheric mantle is not specified explicitly but expressed by a gradual viscosity change, automatically calculated, based on a dry olivine rheology in the mantle and the temperature profile ("Viscous rheology"). The initial temperature profile is prescribed by a three-step linear gradient: $y=0 \mathrm{~km}$ (initial surface level), $T=0^{\circ} \mathrm{C}$; $y=-50 \mathrm{~km}, T=600^{\circ} \mathrm{C} ; y=-150 \mathrm{~km}, T=1,150^{\circ} \mathrm{C}$; and $y=-185 \mathrm{~km}, T=1,300^{\circ} \mathrm{C}$. Temperature boundary conditions are constant temperature at the top and bottom boundaries $\left(T=0^{\circ} \mathrm{C}\right.$ and $T=1,300^{\circ} \mathrm{C}$, respectively) and zero heat flux at the left and right boundaries. Free slip motion boundary condition is prescribed on all boundaries. Left and right boundaries are permeable with constant speed $0.5 \mathrm{~cm} /$ year directed outwards the modeling area. The velocity of the bottom boundary is calculated automatically, so that the mean surface level is kept at $y=0 \mathrm{~km}$. In this example, we apply short-range diffusive erosion only ("Topography") with $k_{s}=50 \mathrm{~m}^{2} /$ year. Model resolution is $2,000 \times 320$ grid nodes and a total number of $6,000,000$ markers is used.

We visualize the accumulated plastic strain in the model in order to distinguish areas of brittle (plastic) and viscous (ductile) deformation in the crust and lithospheric mantle. The upper crust and part of the lower crust are deforming in a brittle regime, while the lowest $15 \mathrm{~km}$ of the lower crust are deforming in a ductile regime; the transformation between these regions is only defined by the rheological parameters, extension rate, and the temperature field. The whole mantle is subject to viscous deformation, with the exception of a very thin $(<10 \mathrm{~km})$ upper layer, which is deforming in a brittle regime.

During the first $30 \mathrm{Myr}$ of extension, the deformation pattern is uniform through the whole crust, yet preserving small-scale fault systems (Fig. 14), which form a landscape similar to the Basin and Range province. After $30 \mathrm{Myr}$, the deformation localizes in two areas, at $x=100 \mathrm{~km}$ and $x=500 \mathrm{~km}$ (Fig. 14b), so that two localized basins begin to develop almost simultaneously.

Figure 15 shows the development of the topography profile with time. The fault system, which results from the extension, tends to increase the topographic variation, while processes of erosion and sedimentation tend to flatten the surface. The balance between these two processes controls the amplitude of the topography,

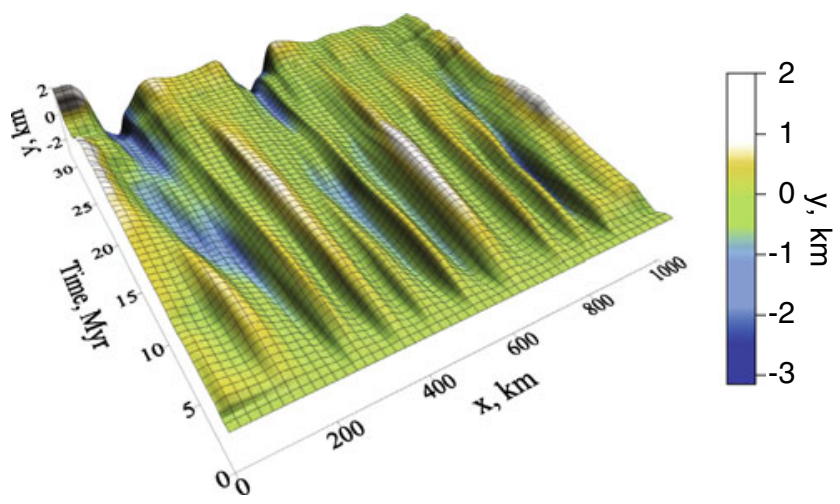

Fig. 15 Topographic time profile of a 1,000-km-width lithospheric area extending at constant speed of $1 \mathrm{~cm} /$ year 
and the lithospheric structure, boundary conditions, and small random fluctuations (including numerical fluctuations) define the pattern of the landscape. Localization of the deformation in the lithosphere results in the formation of two basins and flattering of the rest of the profile after $\sim 25 \mathrm{Myr}$ (Fig. 15).

\section{Basin formation}

As shown in the previous example, extension of unperturbed lithosphere results in an almost uniform deformation in the crust without producing regions where the deformation is significantly more concentrated than in others. The result in ("Lithospheric extension") is observed for cold crust with a Moho temperature $T_{\mathrm{M}}$ of $500^{\circ} \mathrm{C}$ and mafic, strong lower crust. Once deformation in the crust and mantle becomes coupled, it becomes localized, which results in a subsequent narrow basin formation (Buck et al. 1999). However, the period of uniform extension implies that most of the narrow basins formed either after widespread deformation as in the Basin and Range (Bialas and Buck 2009) or that some process localized the deformation in one area from the beginning. Indeed, most continental rift zones form around pre-existing weakness zones within former orogenic belts or at the edges of rheologically strong cratons (Dunbar and Sawyer 1988).

In this example, we consider formation of a narrow basin as a result of lithospheric extension with one preexisting weak fault in the crust (Fig. 16). The model is extended with constant speed $5 \mathrm{~mm} /$ year. Crustal parameters are similar to the ones given in Table 3 , with the following modifications: $\rho_{0}=2,400 \mathrm{~kg} / \mathrm{m}^{3}$ for sediments; upper crustal rheology is anorthosite (Table 2); middle crust has the same parameters as lower crust, except $\rho_{0}=2,800 \mathrm{~kg} / \mathrm{m}^{3} ; H_{r}=10^{-7} \mathrm{~W} / \mathrm{m}^{3}$; plastic weakening in the crust is $80 \%$; and in the prescribed fault, it is $30 \%$ of the normal strength of the rock. The deepening angle of the prescribed fault is $60^{\circ}$.

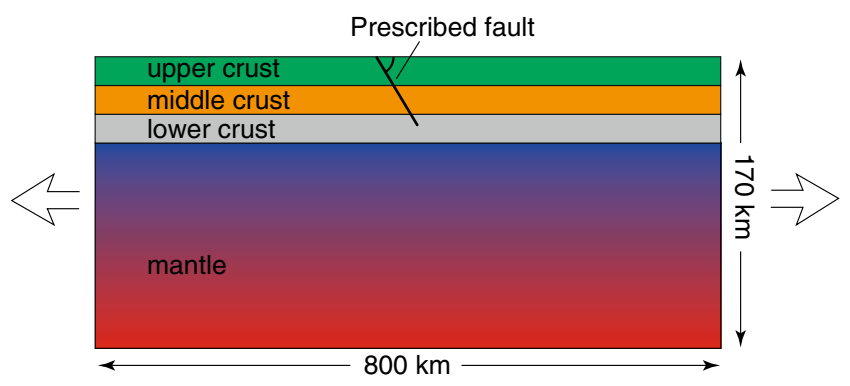

Fig. 16 Model setup for lithospheric extension with prescribed fault
With this example model of basin formation, we discuss the role of the following two parameters, which often do not receive much attention in the numerical models:

- Peierls creep and its role in deformation of the upper lithospheric mantle

- Difference between the models, where in one case sedimentation is applied during the extension and in another case the basin is only filled with sediments during the post-rift phase, after the extension stopped

Peierls creep We perform two numerical experiments: one with Peierls creep in the mantle enabled and another where viscous deformation in the mantle is controlled by diffusion and dislocation creeps only (no Peierls creep). To highlight the effect of Peierls creep, the Moho temperature is set to $550^{\circ} \mathrm{C}$, as the relative influence of this mechanism is the strongest at lower temperatures (Fig. 1). The flat erosion model is applied with $k_{l}=10^{-6}$ year $^{-1}$ and $h_{0}=$ $-200 \mathrm{~m}$ ("Topography"). The lithosphere is extended for 10 Myr (Fig. 17). The model without Peierls creep shows significant faulting in the upper lithospheric mantle while the model with Peierls creep enabled does not.

The lack of Peierls creep causes coupling of deformation between crust and mantle, resulting in more concentrated deformation in the basin area and, consequently, deeper basin. Basin depth in Fig. 17 without Peierls creep is $\sim 11.7 \mathrm{~km}$ whereas it is $\sim 6.8 \mathrm{~km}$ for the model where Peierls creep is included. As expected from Fig. 1, Peierls creep significantly weakens the

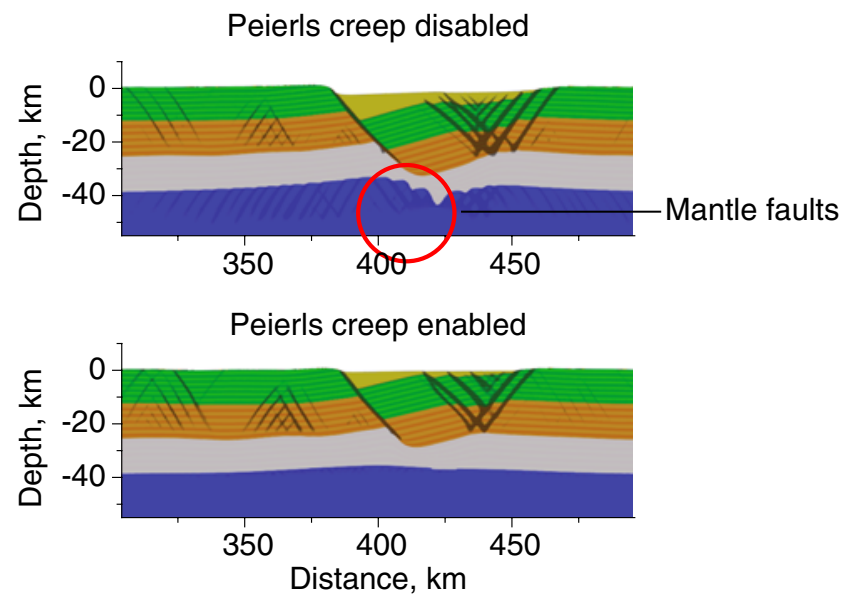

Fig. 17 Deformation in the lithosphere after 10 Myr of extension with one initially prescribed fault in the crust. Two models are shown: (1) Peierls creep in the mantle is disabled (top) and (2) Peierls creep in the mantle is enabled (bottom) 
upper mantle, almost completely eliminating the brittle deformation for $T_{\text {Moho }}>550^{\circ} \mathrm{C}$ and crustal thickness higher than $40 \mathrm{~km}$. Peierls mechanism of deformation can be used to explain the lack of brittle deformation in the upper mantle as an alternative to other weakening mechanisms such as high temperature or hydration.

Syn-rift sedimentation In some numerical models of basin formation, effects of sedimentation are taken into account posteriori, by adding the loading effect of sediments at the last stage of basin formation using an isostatic compensation model (e.g., Lesne et al. 2000). Here we carry out two experiments to find the difference between such models and the models, where processes of sedimentation are applied during the basin formation. The initial model has Moho temperature of $700^{\circ} \mathrm{C}$. The lithosphere is extended with a constant rate of $5 \mathrm{~mm} /$ year during $10 \mathrm{Myr}$, after which extension stops and thermal relaxation takes place. In the first experiment, we do not apply surface processes during the syn-rift stage, but apply flat erosion with $k_{l}=10^{-6}$ year $^{-1}$ and $h_{0}=-200 \mathrm{~m}$ in the post-rift stage, which quickly fills the basin in 2 Myr. In the second experiment, we apply the flat erosion also in the syn-rift stage with the same parameters. By comparing the two cases, we can observe the influence of syn-rift erosionsedimentation alone, since in both cases the basins are completely filled with sediments shortly after extension ceases.

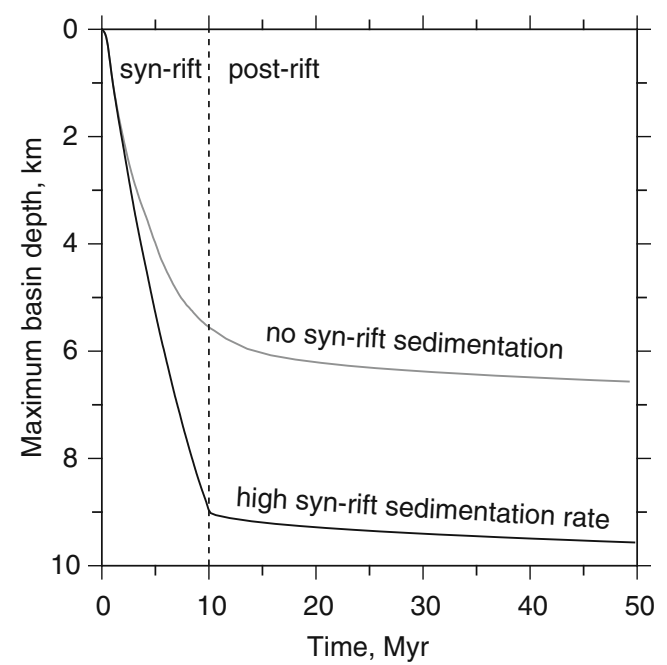

Fig. 18 Maximum depth of the basin vs. time for syn-rift sedimentation example. Two subsidence curves are shown: one with high rate of syn-rift surface processes and one with no syn-rift surface processes. The length of syn-rift stage is $10 \mathrm{Myr}$. High rate of surface processes is applied in both cases in the post-rift stage so that both basins are filled with sediments at that stage
The subsidence curves show that the two basins have different depth (Fig. 18). The basin is $\sim 50 \%$ deeper when syn-rift surface processes are active than without them. The shape of the subsidence curves implies that the changes in the crustal structure imposed by synrift sedimentation are irreversible as the difference in the depth does not decrease with time. The additional subsidence during basin formation can be explained by isostatic compensation in the ductile lower crust for the additional load of syn-rift sediments (cf. Fig. 17). Indeed, the depth to which the crustal block to the right of the prescribed fault will sink is governed by the isostatic forces. However, the movement on the fault is not possible without extension; therefore, sedimentation applied only in the post-rift stage has less effect and results in a smaller final depth of the basin than in the case with active syn-rift surface processes.

\section{Summary}

We present a new thermomechanical code, Samovar, for 2D modeling of lithospheric and sublithospheric processes. It takes into account long- and short-range surface processes, partial melting, and non-linear viscoelastoplastic material deformation. The numerical implementation is based on finite difference discretization on a staggered grid and particle-in-cell approach. We have performed a thorough testing of thermal and mechanical parts of the code.

We apply the code to simulation of lithospheric extension and basin formation. The high resolution that can be obtained with the code on an ordinary PC allows for investigation of very fine features of basin development including individual faults. We show that extension of a uniform lithosphere results in a Basin and Range type surface structure all over the extending area until the deformation pattern between crust and mantle becomes coupled, which results in concentration of deformation around a few narrow basins. A narrow basin can be, however, formed without such coupling if there are preexisting faults in the crust (weakness zones).

Peierls creep significantly weakens the upper lithospheric mantle in the process of basin formation, resulting in viscous-like behavior that produces a smooth Moho uplift. Excluding Peierls creep causes faulting of the upper lithospheric mantle, which results in earlier coupling of deformation between crust and mantle, leading to stress localization and formation of a deeper basin.

We show an importance of sedimentation processes during the extension phase of basin formation because 
the sediment load applied in the syn-rift stage causes irreversible deformation in the crust, which results in a significantly deeper basin than in the case when sedimentation effect is applied posteriori, at the post-rift stage.

\section{References}

Avouac JP, Burov EB (1996) Erosion as a driving mechanism of intracontinental mountain growth. J Geophys Res 101(B8):17747-17769

Babeyko A, Sobolev S (2008) High-resolution numerical modeling of stress distribution in visco-elasto-plastic subducting slabs. Lithos 103:205-216

Bialas RW, Buck WR (2009) How sediment promotes narrow rifting: application to the Gulf of California. Tectonics 28:TC4014. doi:10.1029/2008TC002394

Belytschko T, Liu WK, Moran B (2000) Nonlinear finite elements for continua and structures. Wiley, Chichester

Bittner D, Schmeling H (1995) Numerical modelling of melting processes and induced diapirism in the lower crust. Geophys J Int 123(1):59-70

Blake A (1985) Handbook of mechanics, materials, and structures. Wiley, New York

Braun J, Sambridge M (1997) Modelling landscape evolution on geological time scales: a new method based on irregular spatial discretization. Basin Res 9:27-52

Buck WR, Lavier LL, Poliakov ANB (1999) How to make a rift wide. Philos Trans R Soc Lond Ser A Math Phys Sci 357(1753):671-693. doi:10.1098/rsta.1999.0348

Burov E, Poliakov A (2001) Erosion and rheology controls on synrift and postrift evolution: verifying old and new ideas using a fully coupled numerical model. J Geophys Res Solid Earth 106:16461-16481

Burov E, Toussaint G (2007) Surface processes and tectonics: forcing of continental subduction and deep processes. Glob Planet Change 58(1-4):141-164. doi:10.1016/j.gloplacha. 2007.02.009

Byerlee J (1978) Friction of rocks. Pure Appl Geophys 116: 615-626

Clauser C, Huenges E (1995) Thermal conductivity of rocks and minerals. In: Ahrens $\mathrm{T}$ (ed) Rock physics and phase relations. American Geophysical Union, Washington, DC, pp 105-126

Coulomb C (1773) Test on the applications of the rules of maxima and minima to some problems of statics related to architecture. Mem Math Phys 7:343-382

Cundall P (1989) Numerical experiments on localization in frictional materials. Arch Appl Mech (Ingenieur Archiv) 59(2):148-159

Dunbar JA, Sawyer DS (1988) Continental rifting at pre-existing lithospheric weaknesses. Nature 333:450-452

Evans B, Goetze C (1979) The temperature variation of hardness of olivine and its implication for polycrystalline yield stress. J Geophys Res 84:5505-5524

Fischer KD, Jahr T, Jentzsch G (2004) Evolution of the Variscan foreland-basin: modelling the interactions between tectonics and surface processes. Phys Chem Earth 29(10):665-671. doi:10.1016/j.pce.2004.03.004

Frost H, Ashby M (1982) Deformation-mechanism maps; the plasticity and creep of metals and ceramics. Pergamon, Oxford
Garcia-Castellanos D, Verge's J, Gaspar-Escribano J, Cloetingh S (2003) Interplay between tectonics, climate, and fluvial transport during the cenozoic evolution of the ebro basin (ne iberia). J Geophys Res 108(B7):2347. doi:10.1029/2002JB002073

Gerya T, Yuen D (2003) Rayleigh-Taylor instabilities from hydration and melting propel [']cold plumes' at subduction zones. Earth Planet Sci Let 212(1-2):47-62

Gerya T, Burg J (2007) Intrusion of ultramafic bodies into the continental crust: numerical simulation. Phys Earth Planet Inter 160:124-142

Gerya T, Yuen D (2007) Robust characteristics method for modelling multiphase visco-elasto-plastic thermo-mechanical problems. Phys Earth Planet Inter 163:83-105

Harlow FH, Welch JE (1965) Numerical calculation of timedependent viscous incompressible flow of fluid with a free surface. Phys Fluid 8:2182-2189

Kameyama M, Yuen D, Karato S (1999) Thermal-mechanical effects of low-temperature plasticity (the Peierls mechanism) on the deformation of a viscoelastic shear zone. Earth Planet Sci Lett 168:159-172

Karato S (2008) Deformation of earth materials. Cambridge University Press, Cambridge

Karato S, Wu P (1993) Rheology of the upper mantle: a synthesis. Science 260:771-778

Karato S, Jung H (2003) Effects of pressure on high-temperature dislocation creep in olivine. Philos Mag 83:401-414

Karato S, Paterson M, Fitzgerald J (1986) Rheology of synthetic olivine aggregates-influence of grain size and water. J Geophys Res 91:8151-8176

Karato S, Riedel M, Yuen D (2001) Rheological structure and deformation of subducted slabs in the mantle transition zone: implications for mantle circulation and deep earthquakes. Phys Earth Planet Inter 127:83-108

Katayama I, Karato S (2008) Low-temperature, high-stress deformation of olivine under water-saturated conditions. Phys Earth Planet Inter 168:125-133

Kaus B (2005) Modelling approaches to geodynamic processes. $\mathrm{PhD}$ thesis, ETH Zurich

Kaus BJ, Steedman C, Becker TW (2008) From passive continental margin to mountain belt: insights from analytical and numerical models and application to Taiwan. Phys Earth Planet Inter 171(1-4):235-251. doi:10.1016/j.pepi.2008.06.015

Khan AS, Huang S (1995) Continuum theory of plasticity. Wiley, New York

Kooi H, Beaumont C (1994) Escarpment evolution on highelevation rifted margins: insights derived from a surface processes model that combines diffusion, advection, and reaction. J Geophys Res 99:12191-12209

Lesne O, Calais E, Deverchere J, Chery J, Hassani R (2000) Dynamics of intracontinental extension in the north Baikal rift from two-dimensional numerical deformation modeling. J Geophys Res 105(B9):21727-21744

Mei S, Kohlstedt D (2000a) Influence of water on plastic deformation of olivine aggregates 1 . Diffusion creep regime. J geophys res 105:21457-21469

Mei S, Kohlstedt D (2000b) Influence of water on plastic deformation of olivine aggregates 2. Dislocation creep regime. J Geophys Res 105:21471-21482

Moresi L, Muhlhaus HB (2006) Anisotropic viscous models of large-deformation Mohr-Coulomb failure. Philos Mag 86:3287-3305

Moresi L, Dufour F, Muhlhaus HB (2003) A Lagrangian integration point finite element method for large deformation modeling of viscoelastic geomaterials. J Comput Phys 184: 476-497 
Moresi L, Quenette S, Lemiale V, Meriaux C, Appelbe B, Muhlhaus HB (2007) Computational approaches to studying non-linear dynamics of the crust and mantle. Phys Earth Planet Inter 163:69-82

Ohtani E, Nagata Y, Suzuki A, Kato T (1995) Melting relations of peridotite and the density crossover in planetary mantles. Chem Geol 120(3-4):207-221

Pinkerton H, Stevenson R (1992) Methods of determining the rheological properties of magmas at sub-liquidus temperatures. J Volcanol Geotherm Res 53(1-4):47-66

Poliakov A, Cundall P, Podladckikov Y, Lyakhovsky V (1993) An explicit inertial method for the simulation of viscoelastic flow-an evaluation of elastic effects on diapiric flow in 2-layers and 3-layers models. In: Stone DB Runcorn SK (eds) Flow and creep in the solar system: observations, modeling and theory. Kluwer, Dordrecht, pp 175-195

Popov A, Sobolev S (2008) Slim3d: a tool for three-dimensional thermomechanical modeling of lithospheric deformation with elasto-visco-plastic rheology. Phys Earth Planet Inter 171:55-75

Pysklywec R (2006) Surface erosion control on the evolution of the deep lithosphere. Geology 34(4):225-228. doi:10.1130/G21963.1
Ranalli G (1995) Rheology of the earth, 2nd edn. Chapman \& Hall, London

Ranalli G, Murphy D (1987) Rheological stratification of the lithosphere. Tectonophysics 132(4):281-295

Roscoe K (1970) The influence of strains in soil mechanics, 10th Rankine lecture. Geotechnique 20:129-170

Schenk O, Gartner K (2004) Solving unsymmetric sparse systems of linear equations with PARDISO. Future Gener Comput Syst 20(3):475-487

Selzer C, Buiter SJH, Pfiffner OA (2008) Numerical modeling of frontal and basal accretion at collisional margins. Tectonics 27(3). doi:10.1029/2007TC002169

Stuwe K (1995) Thermal buffering effects at the solidus. Implications for the equilibration of partially melted metamorphic rocks. Tectonophysics 248(1-2):39-51

Turcotte D, Schubert G (2002) Geodynamics. Cambridge University Press, Cambridge

Vermeer P (1990) The orientation of shear bands in biaxial tests. Geotechnique 40:223-226

Zhu G, Gerya TV, Yuen DA, Honda S, Yoshida T, Connolly JAD (2009) Three-dimensional dynamics of hydrous thermal-chemical plumes in oceanic subduction zones. Geochem Geophys Geosys 10(11):Q11,006+. doi:10.1029/2009GC002625 